\title{
Improving the accuracy and efficiency of time-resolved electronic spectra calculations: Cellular dephasing representation with a prefactor
}

\author{
Eduardo Zambrano, Miroslav Šulc, and Jiř́ Vaníčela) \\ Laboratory of Theoretical Physical Chemistry, Institut des Sciences et Ingénierie Chimiques, \\ École Polytechnique Fédérale de Lausanne (EPFL), CH-1015 Lausanne, Switzerland
}

(Dated: 15 November 2021)

Time-resolved electronic spectra can be obtained as the Fourier transform of a special type of time correlation function known as fidelity amplitude, which, in turn, can be evaluated approximately and efficiently with the dephasing representation. Here we improve both the accuracy of this approximation - with an amplitude correction derived from the phase-space propagator - and its efficiency - with an improved cellular scheme employing inverse Weierstrass transform and optimal scaling of the cell size. We demonstrate the advantages of the new methodology by computing dispersed time-resolved stimulated emission spectra in the harmonic potential, pyrazine, and the NCO molecule. In contrast, we show that in strongly chaotic systems such as the quartic oscillator the original dephasing representation is more appropriate than either the cellular or prefactor-corrected methods.

Keywords: semiclassical approximation; phase-space propagator; cellular dynamics; Filinov filtering

\section{INTRODUCTION}

Ultrafast spectroscopy with a time resolution as high as $10^{-15} \mathrm{~s}$ is essential for understanding many quantum dynamical processes in chemical physics ${ }^{1}$ Although short time scales should simplify theoretical studies by requiring shorter simulations, solving the time-dependent Schrödinger equation (TDSE) is challenging even for short times due to the exponential scaling with the number of degrees of freedom. An attractive approach offering a compromise between accuracy and computational efficiency is provided by the semiclassical initial value representation methods, ${ }^{[26}$ which benefit from the ultrafast character of the dynamics not only because of lower computational cost, but also because their accuracy deteriorates with increasing time.

The so-called dephasing representation ${ }^{7}$ (DR), is an efficient initial-value-type semiclassical approximation particularly fitted for calculations of time-resolved electronic spectra $\frac{8[9]}{19}$ The DR improves on a previous method ${ }^{10}$ inspired by the semiclassical perturbation theory of Miller and coworkers ${ }^{11}$ In electronic spectroscopy, the DR and closely related approximations are known as Mukamel's phase averaging method ${ }^{12}$ or Wigner-averaged classical limit, and were used by various authors 13 (17) Shi and Geva $a^{17}$ derived this approximation without invoking the semiclassical propagator - by linearizing ${ }^{\sqrt{18 \mid 19}}$ the path integral quantum propagator. Although the original formulation of the DR pertains to a single pair of potential energy surfaces, the generalization to multiple surfaces, and hence to nonadiabatic dynamics, exists. ${ }^{20}$ The DR has many other applications; the method successfully described, e.g., the local density of states and the transition from the Fermi-Golden-Rule to the Lyapunov regime of fidelity decay 21

\footnotetext{
a)Electronic mail: jiri.vanicek@epfl.ch
}

Yet the most attractive feature of the DR is its efficiency: Motivated by numerical comparisons with other semiclassical methods, $\stackrel{8}{8}$ it has been recently proved analytically 22 that the number of trajectories required for convergence of the DR is independent of the system's dimensionality, Hamiltonian, or total evolution time. The efficiency was further increased in the cellular version of the DR, ${ }^{9}$ which was inspired by Heller's cellular dynamics ${ }^{23}$ and which can significantly reduce the required number of trajectories. The original implementation of the cellular DR (CDR), however, does not converge to the DR in the limit of infinite number of trajectories.

Unlike its efficiency, the accuracy of the DR is not always sufficient. The DR is exact in displaced harmonic oscillators ${ }^{12}$ and often accurate in chaotic systems, ${ }^{77}$ but it breaks down in as simple systems as harmonic oscillators with different force constants. This breakdown can be partially remedied by augmenting the DR with a prefactor, 24 which, however, leads to a much higher computational cost per trajectory and also typically requires more trajectories to achieve convergence.

The first goal of the present paper is to describe a general numerical implementation of the prefactor correction and apply it to the calculation of time-resolved electronic spectra. As the numerical evaluation of the CDR requires, incidentally, the same ingredients as the prefactor correction, the second goal is to combine the advantages of the cellular approach and prefactor correction into a single formula, and show that the resulting method, cellular DR with prefactor (CDRP), is able to increase both the efficiency and accuracy of the DR. Our third goal is presenting a major improvement of the cellularization process by employing the inverse Weierstrass transform of the initial state as the optimal sampling weight instead of the widely used Wigner or Husimi functions, and by correlating the size of the cells with their number and the number of degrees of freedom, which guarantees the 
convergence of the CDR to the original DR in the limit of infinite number of trajectories.

The remainder of the paper is organized as follows: The correlation function approach and the DR approximation for evaluating time-resolved stimulated emission spectra is reviewed in Section II in particular, the DR, its prefactor correction, and its cellular version are deduced. After explaining how the new cellular approach provides optimal choices of the sampling weight and width of Gaussian cells, we derive the CDRP, i.e., a method combining the prefactor correction and cellularization into a single framework. Section III contains several analytical and numerical results testing the theory developed in Section II] while Section IV provides conclusions.

\section{THEORY}

\section{A. Time-resolved stimulated emission: spectrum, time correlation function, and dephasing representation.}

To be specific, we will present the methodology for time-resolved stimulated emission (TRSE); modification to other ultrafast processes is straightforward. Within the electric dipole approximation, time-dependent perturbation theory, and ultrashort pulse approximation, the dispersed ${ }^{25 / 26}$ TRSE spectrum can be computed as a Fourier transform of the following correlation function! $8|9| 25 \mid 26$

$$
\begin{aligned}
C_{\mathrm{TRSE}}(t, \tau)= & E_{\mathrm{pu}}^{2} E_{\mathrm{pr}} \operatorname{Tr}\left[\hat{\rho}_{g}(T) \hat{\mu}_{g e} \hat{U}_{e}(\tau+t)^{-1}\right. \\
& \left.\times \hat{\mu}_{e g} \hat{U}_{g}(t) \hat{\mu}_{g e} \hat{U}_{e}(\tau) \hat{\mu}_{e g}\right] .
\end{aligned}
$$

Here $E_{\mathrm{pu}}$ and $E_{\mathrm{pr}}$ denote the amplitudes of the pump and probe laser pulses, $\hat{\rho}_{g}(T)$ represents the nuclear density operator in the electronic ground state at temperature $T, \hat{\mu}_{i j}$ is the transition dipole moment operator coupling electronic states $i$ and $j, \tau$ stands for the time delay between the pump and probe pulses, and $t$ is the time elapsed after the probe pulse. Finally, $\hat{U}_{j}$ denotes the nuclear quantum evolution operator

$$
\hat{U}_{j}=\exp \left(-i \hat{H}_{j} t / \hbar\right) \quad(j=g, e),
$$

with Hamiltonian $\hat{H}_{j}=\hat{T}+\hat{V}_{j}$ where $\hat{T}$ is the nuclear kinetic energy and $\hat{V}_{j}$ is the $j$ th potential energy surface (PES). In all expressions, the hat denotes operators in the Hilbert space of nuclei.

Within the Franck-Condon approximation and zerotemperature limit, correlation function (1) reduces to

$$
C_{\mathrm{TRSE}}(t, \tau)=E_{\mathrm{pu}}^{2} E_{\mathrm{pr}}\left|\mu_{e g}\right|^{4} f(t, \tau),
$$

where

$$
\begin{aligned}
f(t, \tau) & :=\left\langle\psi_{e}(t, \tau) \mid \psi_{g}(t, \tau)\right\rangle, \\
\left|\psi_{j}(t, \tau)\right\rangle & :=\hat{U}_{j}(t) \hat{U}_{e}(\tau)\left|\Psi_{\text {init }}\right\rangle
\end{aligned}
$$

is a specific time correlation function and the initial state $\left|\Psi_{\text {init }}\right\rangle$ is typically the vibrational ground state of the ground PES. The TRSE spectrum, given by 25

$$
\sigma_{\mathrm{TRSE}}(\omega, \tau) \propto \omega E_{\mathrm{pu}}^{2} E_{\mathrm{pr}}\left|\mu_{e g}\right|^{4} \sigma(\omega, \tau),
$$

is proportional to the so-called wave packet spectrum $\sigma$ obtained $^{27}$ as

$$
\sigma(\omega, \tau)=\operatorname{Re} \int_{0}^{\infty} d t f(t, \tau) e^{i \omega t}
$$

Correlation function (4) for the stimulated emission is a particular example of a more general concept of fidelity amplitude, $\stackrel{28}{\text { defined as }}$

$$
f(t)=\left\langle\Psi_{\text {init }}\left|\hat{U}_{1}(t, 0)^{-1} \hat{U}_{2}(t, 0)\right| \Psi_{\text {init }}\right\rangle,
$$

where $U_{J}\left(t_{2}, t_{1}\right), J=1,2$, is the time evolution operator for a time-dependent Hamiltonian $\hat{H}_{J}(t)$ :

$$
\hat{U}_{J}\left(t_{2}, t_{1}\right)=\mathcal{T} \exp \left[-\frac{i}{\hbar} \int_{t_{1}}^{t_{2}} d t^{\prime} \hat{H}_{J}\left(t^{\prime}\right)\right] \quad(J=1,2),
$$

where $\mathcal{T}$ denotes the time-ordering operator.

Correlation function (4) for TRSE is obtained from the general fidelity amplitude (8) by substituting the following time-dependent Hamiltonians $\hat{H}_{J}(t)$ into Eq. $(9)$ :

$$
\begin{aligned}
& \hat{H}_{1}\left(t^{\prime}\right) \equiv \begin{array}{ll}
\hat{H}_{e} & \text { for } 0 \leq t^{\prime} \leq \tau+t
\end{array} \\
& \hat{H}_{2}\left(t^{\prime}\right) \equiv \begin{cases}\hat{H}_{e} & \text { for } 0 \leq t^{\prime} \leq \tau \\
\hat{H}_{g} & \text { for } \tau \leq t^{\prime} \leq \tau+t\end{cases}
\end{aligned}
$$

Note that $\hat{H}_{2}\left(t^{\prime}\right) \equiv \hat{H}_{g}$ if $\tau=0$.

Besides electronic spectroscopy applications, 13 14 16 16 correlation function (8) proved useful, e.g., in NMR spin echo experiments 59 and in the theories of quantum computation, decoherence,,$\sqrt[30]{ }$ and inelastic neutron scattering! ${ }^{[1]}$ Fidelity amplitude was also used as a measure of the dynamical importance of diabatic, nonadiabatic, or spin-orbit couplings, 20132 and of the accuracy of quantum molecular dynamics on an approximate PES $33 / 34$

In practical calculations, correlation function (8) must usually be approximated, and DR provides an efficient semiclassical approximation.7/13|14|16|17] If we denote by $x^{t}:=\left(q^{t}, p^{t}\right)$ the phase-space coordinates at time $t$ of a point along a classical trajectory of the average $e^{8|12| 24}$ Hamiltonian $H:=\left(H_{1}+H_{2}\right) / 2$, the DR of fidelity amplitude (8) can be written as

$$
f_{\mathrm{DR}}(t)=h^{-D} \int d x^{0} \rho_{W}\left(x^{0}\right) e^{i S_{\mathrm{DR}}\left(x^{0}, t\right) / \hbar},
$$

with

$$
\rho_{W}(q, p) \equiv \int d s\left\langle q-s / 2\left|\hat{\rho}_{\text {init }}\right| q+s / 2\right\rangle e^{i s^{\top} \cdot p / \hbar} .
$$

Here $D$ is the number of degrees of freedom, $\rho_{W}$ denotes the Wigner transform of the initial density operator 
$\hat{\rho}_{\text {init }}=\left|\Psi_{\text {init }}\right\rangle\left\langle\Psi_{\text {init }}\right|$, and $S_{\mathrm{DR}}\left(x^{0}, t\right)$ is the action due to the difference $\Delta H:=H_{2}-H_{1}$ along trajectory $x^{t}$ :

$$
S_{\mathrm{DR}}\left(x^{0}, t\right)=-\int_{0}^{t} d t^{\prime} \Delta H\left(x^{t^{\prime}}, t^{\prime}\right) .
$$

For TRSE, $\Delta H$ is given by

$$
\Delta H \equiv \begin{cases}0 & \text { for } 0 \leq t^{\prime} \leq \tau \\ V_{g}-V_{e} & \text { for } \tau \leq t^{\prime} \leq \tau+t .\end{cases}
$$

Denoting the phase-space average of a quantity $A(x)$ with respect to a weight function $w(x)$ by

$$
\langle A(x)\rangle_{w(x)}:=\frac{\int d x A(x) w(x)}{\int d x w(x)},
$$

time correlation function (11) can be written in a compact way as

$$
f_{\mathrm{DR}}(t)=\left\langle e^{i S_{\mathrm{DR}}\left(x^{0}, t\right) / \hbar}\right\rangle_{\rho_{W}\left(x^{0}\right)} .
$$

Formula (16) can be evaluated efficiently by Monte Carlo integration. Indeed, because the convergence of the DR is independent of dimensionality, the DR is in manydimensional systems much more efficient than other quantum or classical algorithms for computing the fidelity amplitude ${ }^{22}$ The accuracy of the DR typically improves with decreasing $\Delta H$ and increasing complexity of Hamiltonians $\mathrm{H}_{1}$ and $\mathrm{H}_{2}$. While the DR is exact in displaced harmonic oscillators with arbitrary displacement, this perturbative approximation breaks down in some singular cases, such as when Hamiltonians $H_{1}$ and $\mathrm{H}_{2}$ represent harmonic oscillators with significantly different force constants! 12

\section{B. Prefactor correction}

The above-mentioned breakdown of the DR can be partially corrected by including a prefactor in the DR formula $\sqrt{16},{ }^{24}$ We now briefly derive this improved version of the DR.

Fidelity amplitude (8) can be expressed as the expectation value of the echo operator ${ }^{28} \hat{\mathcal{E}}(t):=$ $\hat{U}_{1}(t, 0)^{-1} \hat{U}_{2}(t, 0)$ :

$$
f(t)=\operatorname{Tr}[\hat{\rho} \hat{\mathcal{E}}(t)]=\left\langle\mathcal{E}_{W}\left(x^{0}, t\right)\right\rangle_{\rho_{W}\left(x^{0}\right)},
$$

where $\mathcal{E}_{W}\left(x^{0}, t\right)$ is the Wigner transform of the echo operator. Note that $\hat{\mathcal{E}}(t)$ itself can be interpreted as a single "forward-backward" evolution operator describing propagation driven by $H_{2}$ for time $t$ followed by a propagation driven by $-H_{1}$ from time $t$ to $2 t$. The path labeled by $x_{\mathrm{fb}}^{t}\left(t^{\prime}\right)$ in Fig. 1 is a classical analog of such a forwardbackward propagation.

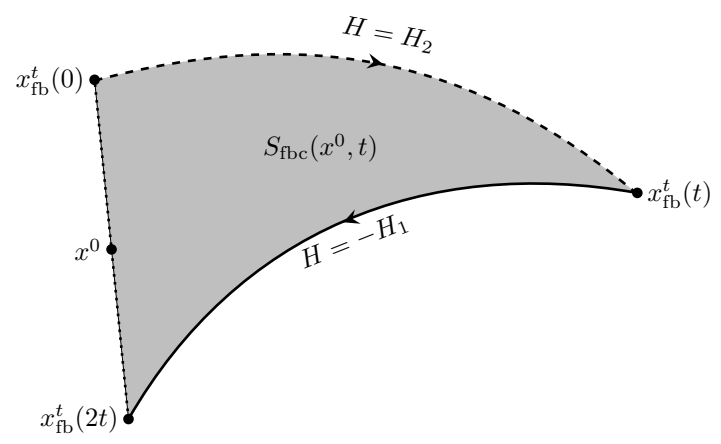

FIG. 1. Sketch of semiclassical evaluation of fidelity amplitude in phase space. Given a phase-space point $x^{0}$, the path $x_{\mathrm{fb}}^{t^{\prime}}$ is determined by two requirements: $(i)$ for $0 \leq t^{\prime} \leq t$ it is driven by $H_{2}$ (dashed path), while for $t \leq t^{\prime} \leq 2 t$ it is driven by $-H_{1}$ (continuous path); and $(i i) x^{0}=\left(x_{\mathrm{fb}}^{0}+x_{\mathrm{fb}}^{t}\right) / 2$. Geometrical part of the phase $S_{\mathrm{fbc}}\left(x^{0}, t\right)$ is the shadowed area and the dotted line is the chord between $x_{\mathrm{fb}}^{0}$ and $x_{\mathrm{fb}}^{t}$.

A semiclassical approximation to the Wigner transform $\mathcal{E}_{W}\left(x^{0}, t\right)$ consists in replacing it by a single phasespace semiclassical propagator,, $35 \mid 36$

$$
\mathcal{E}_{\mathrm{SC}}\left(x^{0}, t\right)=\left|\operatorname{det}\left(I+\frac{J}{2} \cdot \frac{\partial^{2} S_{\mathrm{fbc}}}{\partial\left(x^{0}\right)^{2}}\right)\right|^{\frac{1}{2}} e^{i S_{\mathrm{fbc}}\left(x^{0}, t\right) / \hbar},
$$

with the constraint $x^{0}=\left[x_{\mathrm{fb}}^{t}(2 t)+x_{\mathrm{fb}}^{t}(0)\right] / 2$. Here $I$ is the identity matrix in $2 D$ dimensions and $J$ is the standard symplectic matrix in $2 D$ dimensions,

$$
J=\left(\begin{array}{cc}
0_{D} & I_{D} \\
-I_{D} & 0_{D}
\end{array}\right)
$$

where the subscripts specify the dimensionality of each square block. More details about this semiclassical phase-space propagator are presented in Appendix A. In Eq. (18), phase $S_{\mathrm{fbc}}\left(x^{0}, t\right)$ is the so-called center-action of the path $x_{\mathrm{fb}}^{t}\left(t^{\prime}\right)$ at time $t$; explicitly, this function is defined as

$$
S_{\mathrm{fbc}}\left(x^{0}, t\right):=\oint p^{\top} \cdot d q-\int_{0}^{2 t} d t^{\prime} H\left(x_{\mathrm{fb}}^{t}\left(t^{\prime}\right), t^{\prime}\right),
$$

where the closed integral is evaluated along the path consisting of $x_{\mathrm{fb}}^{t}\left(t^{\prime}\right)$ and of the straight line connecting $x_{\mathrm{fb}}^{t}(2 t)$ and $x_{\mathrm{fb}}^{t}(0)$, as shown in Fig. 1, and

$$
H\left(x_{\mathrm{fb}}^{t}\left(t^{\prime}\right), t^{\prime}\right) \equiv \begin{cases}H_{2}\left(x_{\mathrm{fb}}^{t}\left(t^{\prime}\right), t^{\prime}\right) & \text { for } 0 \leq t^{\prime} \leq t, \\ -H_{1}\left(x_{\mathrm{fb}}^{t}\left(t^{\prime}\right), 2 t-t^{\prime}\right) & \text { for } t \leq t^{\prime} \leq 2 t .\end{cases}
$$

Center-action (20) appears naturally in the Weyl representation of quantum mechanics. ${ }^{36}$ As mentioned in Appendix $\mathrm{A}$, the center-action is a function of the center $x^{0}$ and, in general, is multivalued: a given center $x^{0}$ may be the midpoint between the initial and final points for two or more paths (see, e.g., Fig. 10 in Appendix A). Nevertheless, as shown in Appendix A for our purposes, we can assume that $S_{\mathrm{fbc}}\left(x^{0}, t\right)$ has only a single branch. 
Approximating the center-action in the semiclassical echo operator (18) by the DR action, $S_{\mathrm{fbc}}\left(x^{0}, t\right) \simeq$ $S_{\mathrm{DR}}\left(x^{0}, t\right)$, which is valid up to the first order in perturbation theory, 24373 yields an improved approximation for fidelity amplitude given by $f(t) \approx f_{\mathrm{DRP}}(t)$, where

$$
f_{\mathrm{DRP}}(t)=\left\langle A_{\mathrm{DRP}}\left(x^{0}, t\right) e^{i S_{\mathrm{DR}}\left(x^{0}, t\right) / \hbar}\right\rangle_{\rho_{W}\left(x^{0}\right)},
$$

with

$$
\begin{aligned}
A_{\mathrm{DRP}}\left(x^{0}, t\right) & :=\left|\operatorname{det}\left(I+J \cdot B_{x^{0}}^{t}\right)\right|^{1 / 2}, \\
B_{x^{0}}^{t} & \equiv B\left(x^{0}, t\right):=\frac{1}{2} \frac{\partial^{2} S_{\mathrm{DR}}\left(x^{0}, t\right)}{\partial\left(x^{0}\right)^{2}} .
\end{aligned}
$$

We will refer to expression 222 as the $D R$ with prefactor or DRP: it corresponds to including a prefactor to the contribution of each trajectory in the DR formula (16). The DRP is free of caustics because the prefactor (23) cannot diverge. However, the prefactor is the most expensive part of the DRP evaluation because it depends on the Hessian of the DR phase $S_{\mathrm{DR}}\left(x^{0}, t\right)$ with respect to the initial conditions; in Appendix $\mathrm{B}$ we show how to compute this Hessian from the derivatives of the stability matrix of the classical trajectory. Finally note that switching the PESs in the definition (8) of fidelity amplitude is equivalent to taking the complex conjugate of this equation. DRP preserves this property because of the identity $\operatorname{det}\left(I+J \cdot B_{x^{0}}^{t}\right)=\operatorname{det}\left(I-J \cdot B_{x^{0}}^{t}\right)$, proven in Appendix C.

\section{Cellularization}

The cellular dephasing representation (CDR) was developed in Ref. 9 in order to further accelerate the convergence of the DR in the spirit of Heller's cellular dynamics. ${ }^{23}$ The main idea of the CDR consists in decomposing the Wigner transform of the initial state into phase-space cells and evaluating the contribution of an entire cell of nearby trajectories approximately, using the dynamical information collected along a single, central trajectory. Here we describe a simpler and more rigorous cellularization process than that used in the original CDR (Ref. 9) and other cellularization ${ }^{23138}$ or Filinov filtering 3914 schemes. In particular, the new methodology provides both a natural criterion for cell size [see Eq. 25] ] and a natural sampling weight for the cell centers [given by inverse Weierstrass transform (28)]. Most importantly, unlike the previous approaches, in the limit of infinite number of trajectories, the new methodology converges to the original, noncellular method (in our case, the DR).

In standard cellularization or Filinov filtering procedures, $\frac{923138,41}{41}$ the initial state is covered with phase-space Gaussians as in Fig. 2(a), the centers of these Gaussians being sampled from a given distribution (denoted with a black circle), typically a Wigner or
Husimi transform of the initial state, which is independent of the size and number of cells. Then one decreases the cell size (measured by parameter $\lambda$, defined so that each cell has phase-space volume $\lambda^{2 D} h^{D}$ ) until the approximate treatment of contributions of neighboring trajectories (typically involving quadratic expansion of the action) becomes sufficiently accurate. Independently, the number of cells $N$ is increased until convergence.

There are several problems with this standard approach: First, decreasing the size of the cell to zero $(\lambda \rightarrow 0)$ for a fixed number of cells $N$ eventually results in the initial state not being fully covered [see the middle row of Fig. 2[a)]. Second, in case that the quadratic expansion of the action is accurate, taking the limit $N \rightarrow \infty$ for a fixed nonzero width $\lambda$ is wasteful since many cells are overlapping [see the middle column of Fig. 2(a)]. Third, if the quadratic expansion is inaccurate, taking the limit $N \rightarrow \infty$ for a fixed width $\lambda$ converges to a result different from the original noncellular method. Fourth, for Gaussian initial states and $N=1$, the optimal choice of a single cell is clearly the initial state, but in the standard approach the width and position of the cell are uncorrelated with the number of cells [see the top row of Fig. 2(a)].

The solution of the first three problems is simple and provided by scaling the size of the cell with the number of cells and dimensions according to

$$
\lambda=N^{-1 / 2 D},
$$

guaranteeing that the phase-space volume of the initial state is equal to the total volume of all cells [Fig. 2(b)]. This avoids an ad hoc choice of the width of the cell, replacing two limiting processes $\lambda \rightarrow \infty$ and $N \rightarrow \infty$ with a single process $N \rightarrow \infty$, and pictorially corresponds to going along the diagonal from the top left to the bottom right corner of Fig. 2(a). In the derivation presented below it is shown that the fourth problem is solved by sampling the centers of the cells from the inverse Weierstrass instead of the Wigner transform of the initial state. As we shall see, this inverse Weierstrass transform, represented by red circles in Fig. 2(b) is a natural sampling weight, which is correlated to the size of the cell. If the initial state is a Gaussian, for $N=1$, the single cell has uniquely defined size and position, equal to the size and position of the initial state. In the limit of infinitely many very small cells, their centers are sampled from the Wigner transform. All together, $N$ determines both the size of each cell and the sampling weight for their centers.

To put the above ideas into a precise mathematical form, consider a phase-space Gaussian function centered at the origin,

$$
G_{\Sigma}(x):=\hbar^{D} \sqrt{\operatorname{det} \Sigma} e^{-x^{\top} \cdot \Sigma \cdot x / 2},
$$

where $\Sigma$ is a $2 D \times 2 D$ real, symmetric, positive definite matrix, whose determinant is inversely proportional to the square of the phase-space volume occupied by $G_{\Sigma}$, while the prefactor in Eq. (26) ensures normalization of 


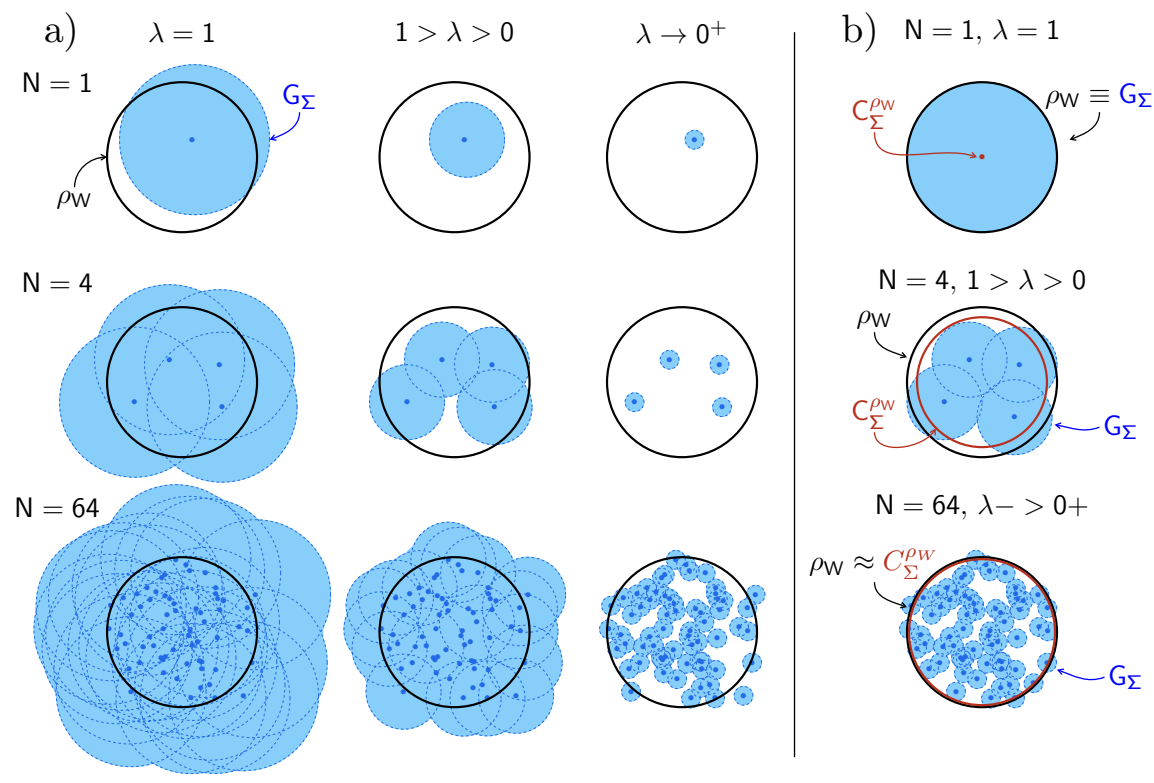

FIG. 2. Comparison of the standard (a) and new (b) cellularization schemes. In both panels, black circles represent the initial state, while the light-blue disks are the Gaussian cells. (a) In the standard procedure, the number of cells $N$ and their size $\lambda \in(0,1]$ are independent. The sampling weight for the cell centers, given by the Wigner function $\rho_{W}$ (black circle), is independent of both $N$ and $\lambda$. (b) In the cellularization procedure proposed in the main text, both the cell size and the sampling weight for their centers are uniquely determined by $N$. The weight, given by inverse Weierstrass transform $C_{\Sigma}^{\rho}{ }^{\prime}$, is denoted with red circles.

$G_{\Sigma}: h^{-D} \int d x G_{\Sigma}(x)=1$. In particular, if $\Sigma_{i, i}=2 / \sigma^{2}$ and $\Sigma_{D+i, D+i}=2 \sigma^{2} / \hbar^{2}$ (for $i=1, \ldots, D$ and $\sigma>0$ ), then $G_{\Sigma}(x)$ coincides with the Wigner transform of a $D$ dimensional Gaussian wave packet with the same width $\sigma$ in all $D$ coordinate directions. However, $G_{\Sigma}(x)$ of Eq. (26) is, in general, not required to be a Wigner transform of any physical quantum state. Most importantly, $G_{\Sigma}(x)$ can be arbitrarily narrow both in position and momentum, and hence does not have to satisfy the Heisenberg uncertainty principle. ${ }^{23}$

Employing sufficiently narrow Gaussian functions (26) with fixed $\Sigma$ as our cells, the Wigner transform of a general state can be expanded as

$$
\begin{aligned}
\rho_{W}(x) & \equiv\left(C_{\Sigma}^{\rho_{W}} * G_{\Sigma}\right)(x) \\
& :=h^{-D} \int d z C_{\Sigma}^{\rho_{W}}(z) G_{\Sigma}(x-z),
\end{aligned}
$$

where the asterisk denotes the convolution of $G_{\Sigma}$ with $C_{\Sigma}^{\rho W}$. Function $C_{\Sigma}^{\rho_{W}}$, playing a role of "continuous expansion coefficient," is known as the inverse Weierstrass transformation of $\rho_{W}$. ${ }^{42}$ Thanks to normalization of $\rho_{W}$ and $G_{\Sigma}$, integrating Eq. (27) over $x$ implies that $C_{\Sigma}^{\rho_{W}}$ is also normalized: $h^{-D} \int d z C_{\Sigma}^{P_{W}}(z)=1$.

Equation (27) can be inverted via the convolution the- orem to obtain

$$
\begin{aligned}
C_{\Sigma}^{\rho_{W}}(z) & =\mathcal{F}^{-1}\left[\mathcal{F}\left[\rho_{W}\right] / \mathcal{F}\left[G_{\Sigma}\right]\right] \\
& \equiv h^{-D} \int d \eta e^{\eta^{\top} \cdot \Sigma^{-1} \cdot \eta / 2 \hbar^{2}} e^{i z^{\top} \cdot \eta / \hbar} \mathcal{F}\left[\rho_{W}\right](\eta),
\end{aligned}
$$

where $\mathcal{F}[\cdot]$ denotes the phase-space Fourier transform,

$$
\mathcal{F}\left[\rho_{W}\right](\eta):=h^{-D} \int d x \rho_{W}(x) e^{-i x^{\top} \cdot \eta / \hbar},
$$

while $\mathcal{F}^{-1}[\cdot]$ stands for its inverse. The Fourier transform of $G_{\Sigma}$ can be evaluated analytically as

$$
\mathcal{F}\left[G_{\Sigma}\right](\eta)=e^{-\eta^{\top} \cdot \Sigma^{-1} \cdot \eta / 2 \hbar^{2}} .
$$

From Eq. 28 we see that $C_{\Sigma}^{\rho_{W}}(z)$ is well-defined only if $\mathcal{F}\left[\rho_{W}\right]$ decays sufficiently faster than $\mathcal{F}\left[G_{\Sigma}\right]$. In other words, the Gaussian cells must be sufficiently narrow in order that the integral (28) over $\eta$ converges.

If the initial state is a Gaussian, i.e., $\rho_{W}(x)=G_{\Sigma^{0}}(x-$ $z^{0}$ ), the cell functions $G_{\Sigma}$ in Eq. 27) can be conveniently chosen as scaled versions of $G_{\Sigma^{0}}$ with widths in all coordinate and momentum directions multiplied by a factor $\lambda$, where $0<\lambda \leq 1$, which is equivalent to setting $\Sigma=\Sigma^{0} / \lambda^{2}$. The width of cell $G_{\Sigma}$ may vary from zero (a delta function) for $\lambda=0$ to the width of the initial state $G_{\Sigma_{0}}$ for $\lambda=1$. The inverse Weierstrass transform (28) can be evaluated analytically for all admissible $\lambda$ (i.e., 
$0 \leq \lambda \leq 1)$ as

$$
C_{\Sigma}^{\rho_{W}}(z)=G_{\Lambda}\left(z-z^{0}\right),
$$

where

$$
\Lambda=\left(1-\lambda^{2}\right)^{-1} \Sigma^{0}=\lambda^{2}\left(1-\lambda^{2}\right)^{-1} \Sigma .
$$

Note that for $\lambda>1$ the inverse Weierstrass transform 28 diverges. The limiting cases of the sampling weight (31) are

$$
C_{\Sigma}^{\rho_{W}}(z)=G_{\Lambda}\left(z-z^{0}\right) \rightarrow \begin{cases}h^{D} \delta\left(z-z^{0}\right), & \lambda=1, \\ G_{\Sigma^{0}}\left(z-z^{0}\right), & \lambda \rightarrow 0^{+},\end{cases}
$$

and are represented, respectively, by the red dot at the top and red circle at the bottom of Fig. 2(b). Indeed, for $\lambda=1$, there is no freedom in the choice of the center of the single cell, whereas in the limit $\lambda \rightarrow 0$, the sampling weight converges to $\rho_{W}$.

Inserting the cellular expansion (27) into the DR formula (16) yields

$f_{\mathrm{DR}}(t)=h^{-2 D} \int d z^{0} C_{\Sigma}^{\rho W}\left(z^{0}\right) \int d x^{0} G_{\Sigma}\left(x^{0}-z^{0}\right) e^{i S_{\mathrm{DR}}\left(x^{0}, t\right) / \hbar}$.

In order to carry out the integration over $x^{0}$ analytically, one expands the DR phase about point $z^{0}$ as $S_{\mathrm{DR}}\left(x^{0}, t\right) \approx$ $S_{\mathrm{CDR}}\left(x^{0}, t ; z^{0}\right)$, where the CDR action is

$S_{\mathrm{CDR}}\left(x^{0}, t ; z^{0}\right):=S_{\mathrm{DR}}\left(z^{0}, t\right)+\delta x^{\top} \cdot \alpha_{z^{0}}^{t}+\delta x^{\top} \cdot B_{z^{0}}^{t} \cdot \delta x$.

In the last equation, $\delta x:=x^{0}-z^{0}, \alpha_{z^{0}}^{t}:=\partial S_{\mathrm{DR}}\left(z^{0}\right) / \partial z^{0}$ is the gradient of $S_{\mathrm{DR}}$ at $z^{0}$, and $B_{z^{0}}^{t^{2}}$, already defined in Eq. (24), is, up to a factor $1 / 2$, the Hessian of $S_{\mathrm{DR}}$ at $z^{0}$. Using the quadratic expansion (35), the integral over $x^{0}$ in the double integral representation (34) of the DR is performed analytically to yield the final result-CDR:

$$
f_{\mathrm{CDR}}(t)=\left\langle A_{\mathrm{CDR}}\left(z^{0}, t\right) e^{i S_{\mathrm{DR}}\left(z^{0}, t\right) / \hbar}\right\rangle_{C_{\Sigma}^{\rho}{ }^{\rho}\left(z^{0}\right)},
$$

with

$$
\begin{aligned}
A_{\mathrm{CDR}}\left(z^{0}, t\right) & :=\left|\operatorname{det}\left(\Sigma \cdot K_{z^{0}}^{t}\right)\right|^{1 / 2} e^{-\left(\alpha_{z^{0}}^{t}\right)^{\top} \cdot K_{z^{0}}^{t} \cdot \alpha_{z^{0}}^{t} / 2 \hbar^{2}}, \\
\Sigma \cdot K_{z^{0}}^{t} & =\left(I-2 i B_{z^{0}}^{t} \cdot \Sigma^{-1} / \hbar\right)^{-1} .
\end{aligned}
$$

Straightforward numerical implementation evaluates $f_{\mathrm{CDR}}(t)$ in Eq. (36) by Monte Carlo importance sampling. This means arithmetically averaging the estimator $A_{\mathrm{CDR}} \exp \left(i S_{\mathrm{DR}} / \hbar\right)$ over the set of $N$ initial conditions sampled from the weight $C_{\Sigma}^{\rho_{W}}$ using the Box-Muller algorithm for Gaussian initial states or Metropolis algorithm for general states. [The positivity of $C_{\Sigma}^{\rho_{W}}$ is for Gaussian initial states guaranteed by Eq. (31).] Equivalently, one can think of this procedure as expanding the Wigner transform $\rho_{W}$ of the initial state into a finite set of Gaussians, i.e.,

$$
\rho_{W}(x) \approx \sum_{n=1}^{N} C_{n} G_{\Sigma}\left(x-z_{n}\right),
$$

where $C_{n}=1 / N$ and centers $\left\{z_{n}\right\}$ are sampled from $C_{\Sigma}^{\rho_{W}}(z)$. This expansion is then combined with the quadratic expansion (35) of $S_{\mathrm{DR}}$ and substituted into the DR formula (11).

As mentioned above, a natural value of the scaling parameter is $\lambda=N^{-1 / 2 D}$ for which the $N$ cells $G_{\Sigma^{0} / \lambda^{2}}$ cover essentially the same phase-space volume as the initial state $\rho_{W}(x)=G_{\Sigma^{0}}\left(x-z^{0}\right)$. Moreover, for $N=1$, Eq. 25) gives $\lambda=1$. From Eq. (33) we see that $C_{\Sigma}^{\rho_{W}}(z)$ degenerates to a delta function and the single cell is identical to $\rho_{W}$. On the other hand, $N \rightarrow \infty$ implies $\lambda \rightarrow 0^{+}$ and Eq. (38) yields $\Sigma \cdot K_{z^{0}}^{t} \rightarrow I$ and $K_{z^{0}}^{t} \rightarrow 0$. Since for $\lambda \rightarrow 0^{+}, C_{\Sigma}^{\rho_{W}}(z) \rightarrow G_{\Sigma^{0}}\left(z-z^{0}\right) \stackrel{z^{0}=}{=} \rho_{W}(z)$ and $A_{\mathrm{CDR}} \rightarrow 1$, comparison of Eqs. (36) and (16) confirms that the CDR reduces in the limit $N \rightarrow \infty$ to the original DR, as promised. Note that this desirable property was satisfied neither by the original CDR nor by standard cellularization or Filinov filtering procedures for the Van Vleck or Herman-Kluk propagators.

Several further improvements are possible: First, a significant boost in computational efficiency could be gained with ideas implemented in the generalized Filinov filtering $40 \mid 41$ or stationary phase Monte Carlo method 43 Motivated by the generalized Filinov method, for instance, one would add a complex linear term to the exponent of the Gaussian cell to ensure that the overall phase of the integrand of the $x^{0}$ integral in Eq. (34) were approximately stationary, making the original integral more amenable to Monte Carlo integration. This is in contrast to the original Filinov approach, $\frac{[39}{,}$ which does not employ an additional phase. Another improvement relies on Sobol sampling, ${ }^{44}$ which actively seeks different initial conditions while preserving the normal distribution, and was used, e.g., by Walton and Manolopoulos $\frac{38}{31}$ Finally, it is advantageous to allow the expansion coefficients $C_{n}$ in Eq. (39) to differ from $1 / N$. Specifically, one finds the optimal coefficients $C_{n}$ for given, already sampled, Gaussian centers $\left\{z_{n}\right\}$ by minimizing the residual $L^{2}$ error of the expansion (39) under the constraints

$$
\begin{aligned}
& \sum_{n=1}^{N} C_{n}=1 \text { and } \\
& C_{n} \geq 0, n=1, \ldots, N,
\end{aligned}
$$

which guarantee that $f_{\mathrm{CDR}}(0)=1$ and $\left|f_{\mathrm{CDR}}(t)\right| \leq 1$. From numerical point of view, this amounts to solving a convex quadratic program ${ }^{45}$ As demonstrated in Sec. III. this procedure further enhances efficiency, nevertheless the acceleration due to the cellularization procedure itself is dominant.

In practice, one should always use all five "tricks," i.e., sampling (36) from the inverse Weierstrass transform, scaling (25) of the cells with $N$, generalized Filinov

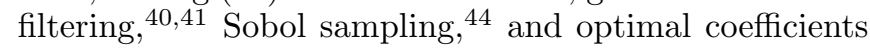
40a-40b) . Although clearly beneficial, generalized Filinov filtering and Sobol sampling were not employed here, in order to clearly separate the effect of the three new ideas presented: sampling (36) from the inverse Weier- 
strass transform, scaling 25 of the cells with $N$, and optimal coefficients 40a)- 40b).

\section{Cellular DR with prefactor correction}

The numerical prerequisites of the CDR (Subsec. II C) and DRP (Subsec. IIB) are the same - the cost per trajectory is determined by evaluating the Hessian of $S_{\mathrm{DR}}$ with respect to initial conditions. This allows for a straightforward combination of the methods, without increasing the cost per trajectory, by multiplying the contribution 36 of each trajectory with the prefactor 23) and thus obtaining the cellular dephasing representation with prefactor (CDRP):

$$
f_{\mathrm{CDRP}}(t)=\left\langle A_{\mathrm{CDRP}}\left(z^{0}, t\right) e^{i S_{\mathrm{DR}}\left(z^{0}, t\right) / \hbar}\right\rangle_{C_{\Sigma}^{\rho W}\left(z^{0}\right)},
$$

where

$$
A_{\mathrm{CDRP}}\left(z^{0}, t\right):=A_{\mathrm{DRP}}\left(z^{0}, t\right) A_{\mathrm{CDR}}\left(z^{0}, t\right) .
$$

In principle, the CDRP should benefit both from the enhanced efficiency of the CDR and improved accuracy of the DRP, as depicted in Fig. 3 .

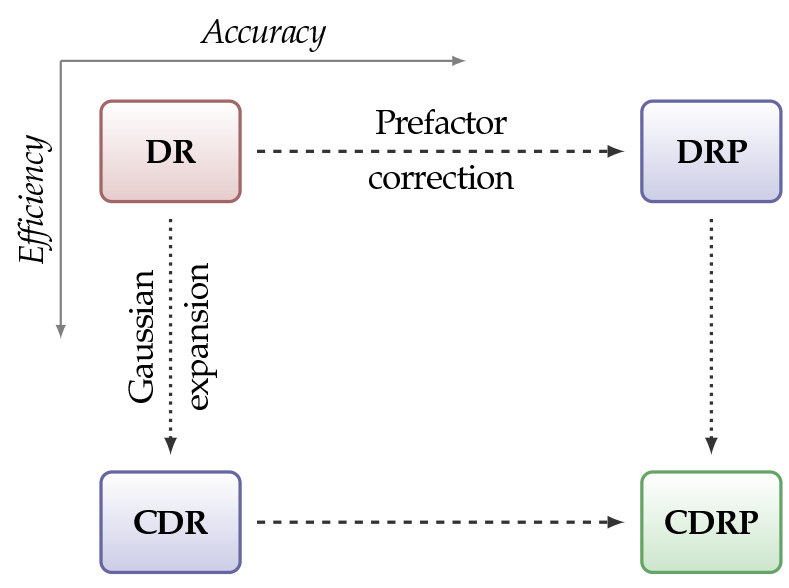

FIG. 3. Relations between several approximations for time correlation function (8). Typically, the accuracy increases along the horizontal arrows, corresponding to adding the prefactor 22, while the efficiency improves in the downward direction, corresponding to the cellularization procedure 36 .

As for the asymptotic computational complexity of Eq. (41) per trajectory, a straightforward implementation scales with system's dimensionality $D$ and total propagation time $t$ as $\mathcal{O}\left(D^{3} t\right)$. Linear scaling with time is easily verified by direct inspection of Eq. 41, while the cubic dependence on $D$ is due to the necessity to propagate the stability matrix and due to the matrix operations implicit in Eqs. (36) and (37). The CDRP is thus cheaper than, e.g., the popular Forward Backward Initial Value Representation ${ }^{46}$ which would scale as $\mathcal{O}\left(D^{3} t^{2}\right)$.

\section{NUMERICAL EXAMPLES}

In this section we will show how the CDRP approximation improves the accuracy of the time correlation function (8) and stimulated emission spectrum (7) for several well-known systems.

\section{A. Harmonic oscillators}

As the first example we consider two quadratic Hamiltonians in $D$ dimensions:

$$
\begin{aligned}
H_{g} & =\frac{1}{2} x^{\top} \cdot \mathcal{H}_{g} \cdot x, \\
H_{e} & =\frac{1}{2}(x-d)^{\top} \cdot \mathcal{H}_{e} \cdot(x-d)+V_{0},
\end{aligned}
$$

where $V_{0}$ is the gap between the two potential wells,

$$
\mathcal{H}_{j}:=\frac{\partial^{2} H_{j}}{\partial x^{2}}=\left(\begin{array}{cc}
k_{j} & 0_{D} \\
0_{D} & m^{-1}
\end{array}\right) \quad(j=g, e),
$$

is the $2 D \times 2 D$ Hessian matrix of $H_{j}, k_{j}$ being the forceconstant matrix, $m_{i j}=m_{i} \delta_{i j}$ is the $D \times D$ matrix of masses, and $d=\left(d_{q}, d_{p}\right)$ is the phase-space displacement of the two Hamiltonians: e.g., $d_{q}$ is the coordinate distance between the two potential minima. The Hessian of the average Hamiltonian is given by the (invertible) $2 D \times 2 D$ matrix

$$
\mathcal{H}:=\frac{\partial^{2} H}{\partial x^{2}}=\left(\begin{array}{cc}
k & 0_{D} \\
0_{D} & m^{-1}
\end{array}\right),
$$

where $k:=\left(k_{g}+k_{e}\right) / 2$. The path driven by the average Hamiltonian is

$$
x^{t}=M^{t} \cdot\left(x^{0}-\delta\right)+\delta,
$$

where $M^{t}:=\exp (t J \cdot \mathcal{H})$ is the stability matrix for $\mathcal{H}$ and $\delta:=\mathcal{H}^{-1} \cdot \mathcal{H}_{e} \cdot d / 2$. Since the Hamiltonians 43 are quadratic, it is possible to evaluate the DR phase analytically for an arbitrary initial condition $x^{0}$ as

$$
S_{\mathrm{DR}}\left(x^{0}, t\right) \equiv\left(x^{0}-\delta\right)^{\mathrm{\top}} \cdot B^{t} \cdot\left(x^{0}-\delta\right)+\left(x^{0}-\delta\right)^{\top} \cdot v^{t}+a^{t},
$$

where

$$
\begin{aligned}
B^{t} & \equiv-\frac{1}{2} \int_{0}^{t} d t^{\prime}\left(M^{t^{\prime}}\right)^{\top} \cdot \Delta \mathcal{H} \cdot M^{t^{\prime}} \\
v^{t} & :=-2 \int_{0}^{t} d t^{\prime}\left(M^{t^{\prime}}\right)^{\top} \cdot\left(\mathcal{H}+\frac{\Delta \mathcal{H}}{2}\right) \cdot \delta, \\
a^{t} & :=\left(V_{0}+\frac{1}{2} \delta^{\top} \cdot \Delta \mathcal{H} \cdot \delta_{+}\right) t
\end{aligned}
$$

with $\Delta \mathcal{H}:=\mathcal{H}_{g}-\mathcal{H}_{e}$ and $\delta_{+}:=\mathcal{H}^{-1} \cdot \mathcal{H}_{g} \cdot d / 2$. Note that in the harmonic systems, the cellular schemes are exactly equal to their noncellular analogs, e.g.,

$$
f_{\mathrm{CDR}}(t) \equiv f_{\mathrm{DR}}(t)=\left\langle e^{i S_{\mathrm{DR}}\left(x^{0}, t\right) / \hbar}\right\rangle_{\rho_{W}\left(x^{0}\right)} .
$$


[However, if a discrete Gaussian expansion (39) is used, the accuracy of the results will be limited by the error inherent in Eq. (39).] Since $B^{t}$ and hence $A_{\mathrm{DRP}}(t)$ are in this case independent of $x^{0}$, the DRP and CDRP can be calculated for an arbitrary initial state as

$$
f_{\mathrm{CDRP}}(t) \equiv f_{\mathrm{DRP}}(t) \equiv A_{\mathrm{DRP}}(t) f_{\mathrm{DR}}(t)
$$

Explicit formulas for one degree of freedom are

$$
\begin{aligned}
& B^{t}=-\Delta k\left(\begin{array}{cc}
t+\sin (2 \omega t) / 2 \omega & \sin ^{2}(\omega t) / m \omega^{2} \\
\sin ^{2}(\omega t) / m \omega^{2} & \frac{t}{(m \omega)^{2}}-\frac{\sin (2 \omega t)}{2 \omega(m \omega)^{2}}
\end{array}\right), \\
& v^{t}=\left(1-\frac{\Delta k}{2 m \omega^{2}}\right)^{2}\left(\begin{array}{l}
m \omega \sin (\omega t) \\
1-\cos (\omega t)
\end{array}\right), \\
& a^{t}=V_{o} t+\frac{d^{2}}{8} \Delta k\left[1-\left(\frac{\Delta k}{2 m \omega^{2}}\right)^{2}\right] t .
\end{aligned}
$$

Here, $\omega^{2}:=k / m, \Delta k:=k_{g}-k_{e}$, and $d_{p}=0$, i.e., $d$ has only position components. Additionally, the determinant prefactor is given by

$$
A_{\mathrm{DRP}}(t)=\left|1+\left(\frac{\Delta k}{4 m \omega}\right)^{2}\left(t^{2}-\frac{\sin ^{2} \omega t}{\omega^{2}}\right)\right|^{\frac{1}{2}} .
$$

Figure 4 shows the fully converged time correlation functions for zero time delay in one-dimensional harmonic oscillator 43 using a Gaussian initial state. We observe the effect of the prefactor (23): it enhances the accuracy compared with the DR, so that the approximate time correlation function does not decay with increasing time. Note that the Fourier transforms of time correlations shown in Fig. 4 can be interpreted both as TRSE spectra with zero time delay and as continuous-wave $a b$ sorption spectra.

Now we consider a two-dimensional harmonic system (43) with $d_{q}=\left(d_{1}, 0\right), d_{p}=(0,0)$,

$$
k_{g}=\left(\begin{array}{cc}
k_{1} & 0 \\
0 & k_{1}
\end{array}\right), \quad \text { and } \quad k_{e}=\left(\begin{array}{cc}
k_{1} & 0 \\
0 & k_{2}
\end{array}\right) \text {, }
$$

which is a prototype of the breakdown of the DR in simple molecular systems. While the DR describes exactly the behavior of the "excited" mode corresponding to displaced simple harmonic oscillators,$\frac{12}{2}$ this agreement is lost due to the decay of the DR in the "silent" mode, corresponding to harmonic oscillators with different force constants [as in Fig. 4(a)], in which the DR breaks down. In other words, the breakdown of the DR for the uninteresting mode covers up the accurate information about the interesting mode. Figure 5 shows the time correlation function for time delay $\tau=10$, confirming that the DRP can in this system almost completely remove the error introduced by the DR.

\section{B. Pyrazine model}

The next system is based on the four-dimensional vibronic coupling model taking into account normal modes $\nu_{1}, \nu_{6 \mathrm{a}}, \nu_{9 \mathrm{a}}$, and $\nu_{10 \mathrm{a}}$ of pyrazine ${ }^{47}$ We employ the $S_{0}$ and $S_{1}$ surfaces from Ref. 47, but disregard the nonadiabatic coupling between states $S_{1}$ and $S_{2}$ since for the $S_{0} \rightarrow S_{1}$ excitation this coupling is much less important than for the $S_{0} \rightarrow S_{2}$ excitation and since nonadiabatic dynamics is not our primary focus. However, even this simplified model requires a nontrivial Duschinsky rotation ${ }^{48}$ connecting normal modes of the $S_{0}$ and $S_{1}$ states.

Since the pyrazine model is globally quadratic, the action expansion in Eq. (35) is exact (as discussed in Subsec. III A and thus the fully converged DR and DRP correlation functions can be obtained by the cellular variants $\mathrm{CDR}_{N=1}$ and $\mathrm{CDRP}_{N=1}$ of these methods obtained with a single trajectory.

Figure 6(a) shows pyrazine TRSE correlation function $f(t, \tau)$, calculated for a particular delay time $\tau \approx$ 48 fs and multiplied by a phenomenological damping function ${ }^{49}$

$$
\chi(t):=\cos ^{2}[\pi t /(2 T)] \theta(T-t),
$$

where $T$ denotes the total propagation time. Parameters of the calculation are summarized in the caption of Fig. 6] The DRP is shown in Fig. 6(a) to yield an excellent agreement with the quantum calculation. This is also confirmed in the corresponding spectrum [Fig. 6(b)], computed as the Fourier transform (7) of the damped correlation function.

Finally, Fig. 6(c) compares the convergence behavior of individual methods. The convergence is quantified by the relative $L^{2}$ error achieved for $N \ll N_{\text {ref }}$ trajectories:

$$
\eta\left(N, N_{\text {ref }}\right):=\left\|\left(f_{N}-f_{N_{\text {ref }}}\right) \chi\right\| /\left\|f_{N_{\text {ref }}} \chi\right\|,
$$

where $\|f\|^{2}:=\int_{0}^{T} d \tau^{\prime}\left|f\left(\tau^{\prime}\right)\right|^{2}$. The subscript $N$ of $f_{N}$ in Eq. (57) emphasizes that the quantity $f_{N}$ was computed with $N$ trajectories, while the fully converged results are denoted by $N \rightarrow \infty$. Time integrals appearing implicitly in Eq. (57) are evaluated with Simpson's method. The cellularization accelerates convergence by lowering the number of trajectories required to achieve the same statistical error by about two orders of magnitude [Fig. 6(c)]. Additional minor improvement is achieved by optimizing the expansion coefficients in Eq. (39) using constraints 40.

\section{Quartic oscillator}

After discussing harmonic systems, which are rather simple even in high dimensions, let us turn to the opposite limit of chaotic dynamics, which can present difficulties even in few dimensions. In particular, we consider a two-dimensional chaotic quartic oscillator. 50 The two 


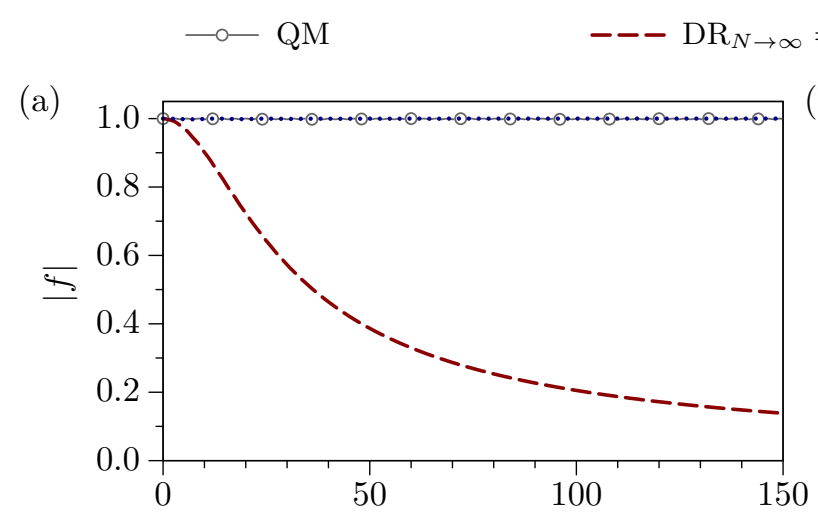

$=\mathrm{CDR}_{N=1}$

$\operatorname{DRP}_{N \rightarrow \infty}=\operatorname{CDRP}_{N=1}$
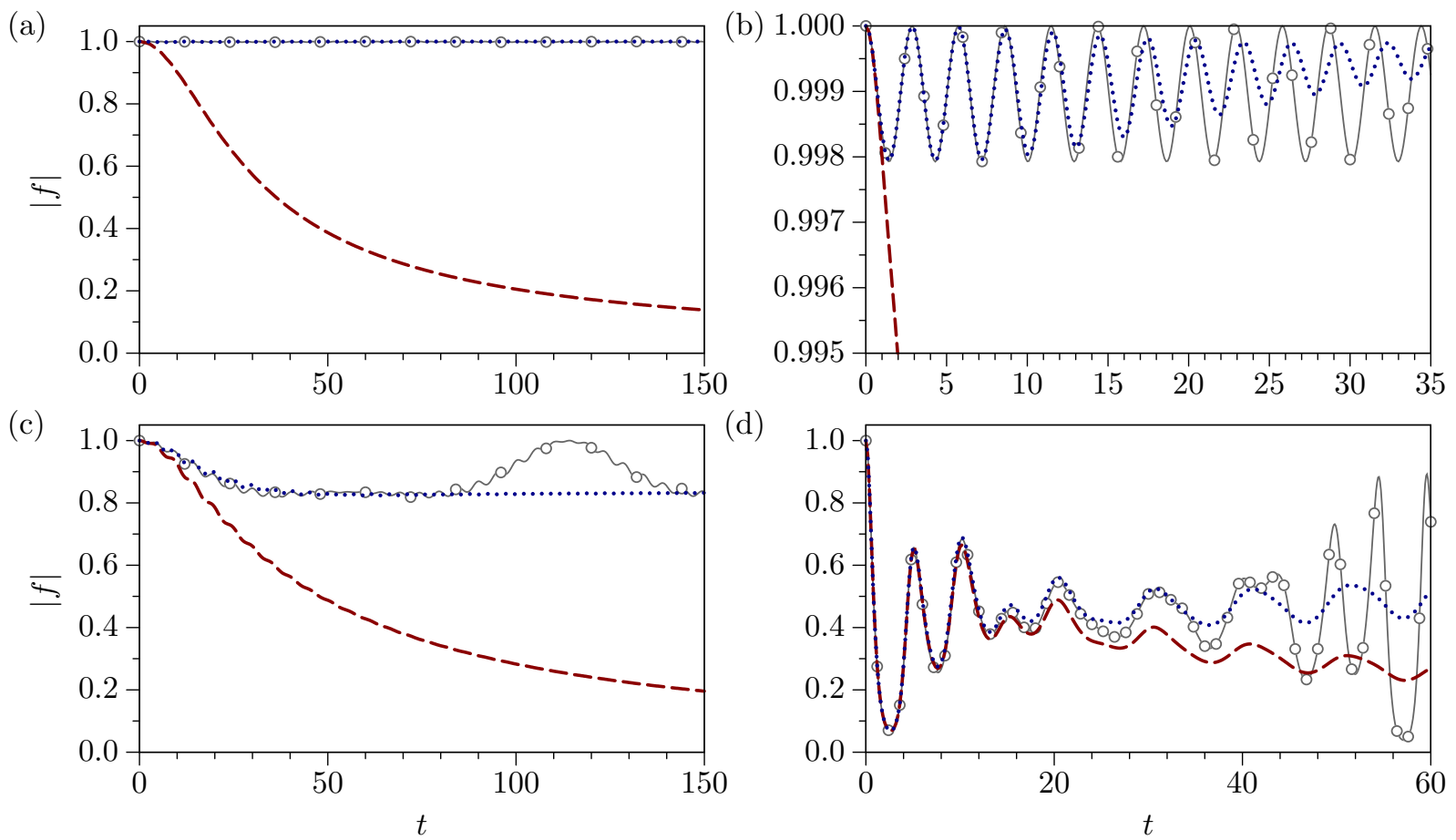

(d)

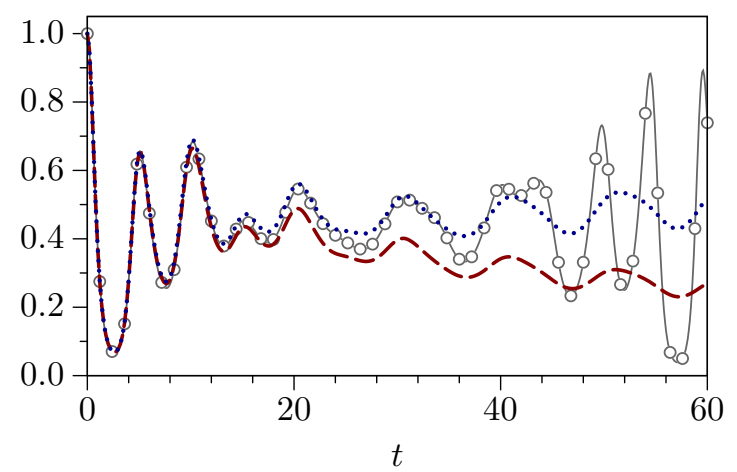

FIG. 4. Time correlation functions for time-resolved stimulated emission spectrum with a zero time delay $(\tau=0)$ in a onedimensional harmonic potential (43) with $V_{0}=0$, force constants $k_{g}=1$ and $k_{e}=1.2$. The initial state is a Gaussian wave packet with width $\sigma$ and centered at $z^{0}$. (a) $m=1, \sigma=1, z^{0}=(0,0)$, and $d=(0,0)$. (b) Detail of panel (a). (c) $m=3$, $\sigma=1, z^{0}=(0.3,0.3)$, and $d=(0,0)$. (d) $m=3, \sigma=2, z^{0}=(0.5,0)$, and $d=(1,0)$.

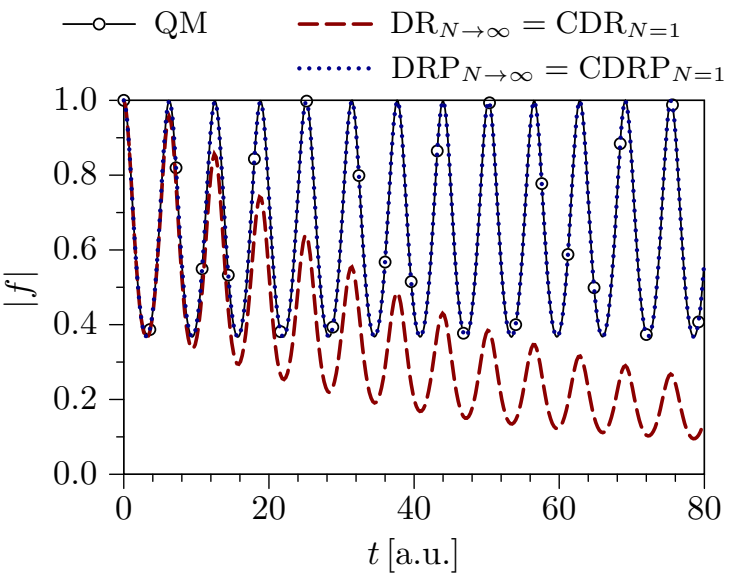

FIG. 5. Time correlation function for time-resolved stimulated emission spectrum in a two-dimensional harmonic oscillator model. Displacements are $d=\left(d_{q}, d_{p}\right)$ with $d_{q}=(1,0)$ and $d_{p}=(0,0), V_{0}=10$, and $m=1$, and force constants [according to Eq. [55] ] are $k_{1}=1$ and $k_{2}=2$. The initial state is the ground state of the ground PES. Time delay $\tau=10$.

potential energy surfaces,

$$
V_{j}\left(q_{1}, q_{2}\right)=\frac{q_{1}^{2} q_{2}^{2}}{2}+\frac{\beta_{j}}{4}\left(q_{1}^{4}+q_{2}^{4}\right)
$$

differ only in the parameter $\beta_{j}>0$. Chaotic behavior is due to the coupling term $q_{1}^{2} q_{2}^{2} / 2$ since in the limit $\beta_{j} \rightarrow$ $\infty$, the Hamiltonian $T+V_{j}$ becomes separable and hence integrable.

Due to the chaotic character of this system, one expects that the central ingredient of the cellularization, i.e., the quadratic expansion of the action difference in Eq. (35) will be poor and hinder convergence. This is indeed confirmed in Fig. 7(a), showing the difference of the DR action (13) for two neighboring trajectories specified by initial conditions $z^{0}$ and $w^{0}$, i.e.,

$$
\delta S_{\mathrm{DR}}(t):=S_{\mathrm{DR}}\left(w^{0}, t\right)-S_{\mathrm{DR}}\left(z^{0}, t\right) .
$$

This quantity is then compared with predictions based on the quadratic expansion (35) and its linear part. The expansion order denoted "linear $+1 / 2$ " is a widely used approximation 23138 to the quadratic expansion (35) within which one neglects the third derivatives of the potential (see Appendix B). Figure 7(a) shows clearly that in the quartic oscillator the quadratic expansion (35) is reliable only for short times and that the linear expansion is superior to the presumably more accurate "linear $+1 / 2$ " approach.

As a consequence, Fig. 8, comparing the TRSE correlation functions, shows that the method of choice for the quartic oscillator is the "bare" DR [Fig. 8(a)], since the CDR [Fig. 8(c)] converges more slowly, while the DRP 

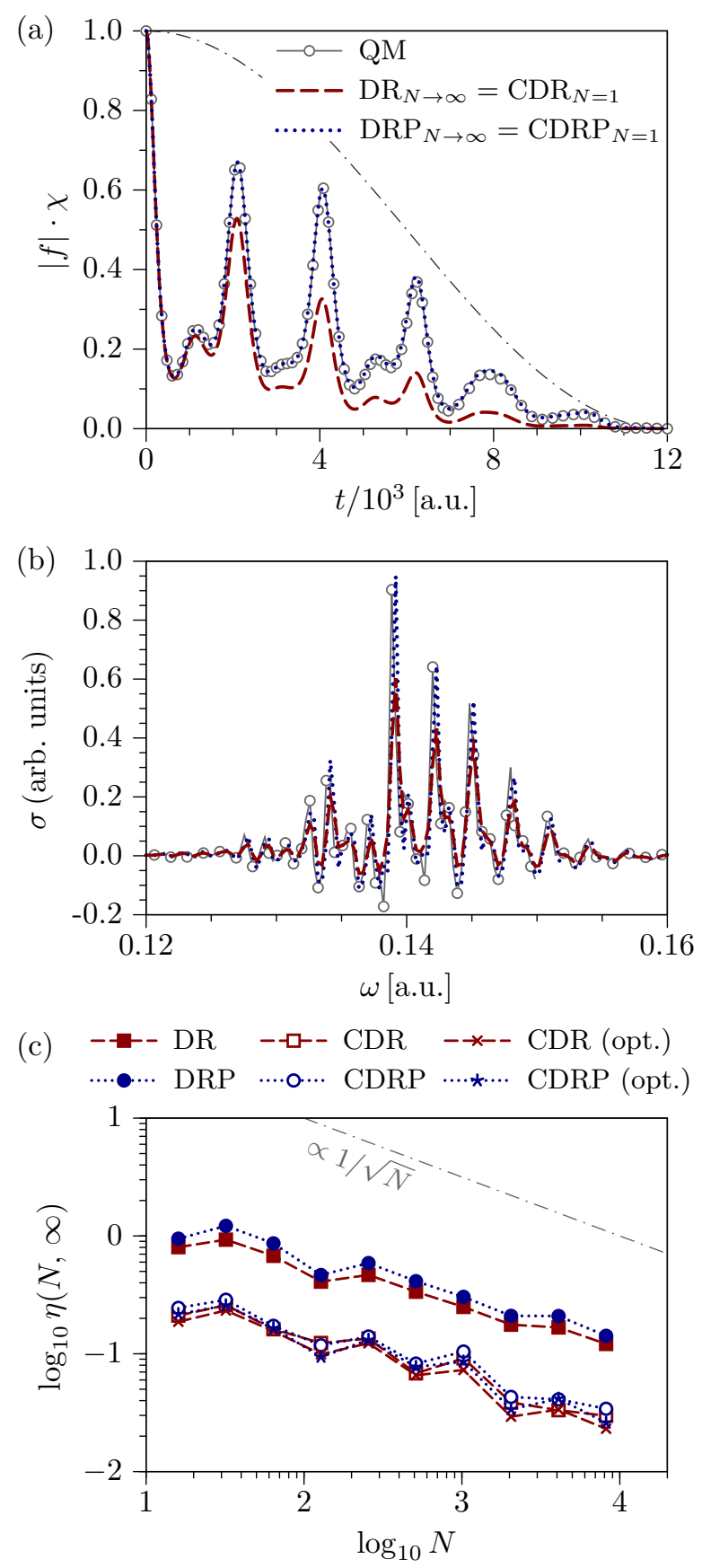

FIG. 6. Time-resolved stimulated emission in the pyrazine model of Subsec. IIIB Initial state is the ground state of the $S_{0}$ surface, the delay time $\tau=2 \times 10^{3}$ a.u. $\approx 48 \mathrm{fs}$. (a) Time correlation function [damped by $\chi(t)$ of Eq. (56), shown as a dash-dotted line]. (b) Corresponding spectrum. (c) Convergence error $\eta$ [defined in Eq. (57)] of the damped correlation function as a function of the number of trajectories $N$. The points labeled by "opt." were computed with optimized expansion coefficients $C_{n}$ of Eq. 40 (see Subsec. II C).
(a)
$\delta S_{\mathrm{DR}}$ linear $\delta S_{\mathrm{DR}}$ $\cdots \cdots \cdots S_{\mathrm{DR}}$ linear $+1 / 2$
-.-. $\delta S_{\mathrm{DR}}$ quadratic
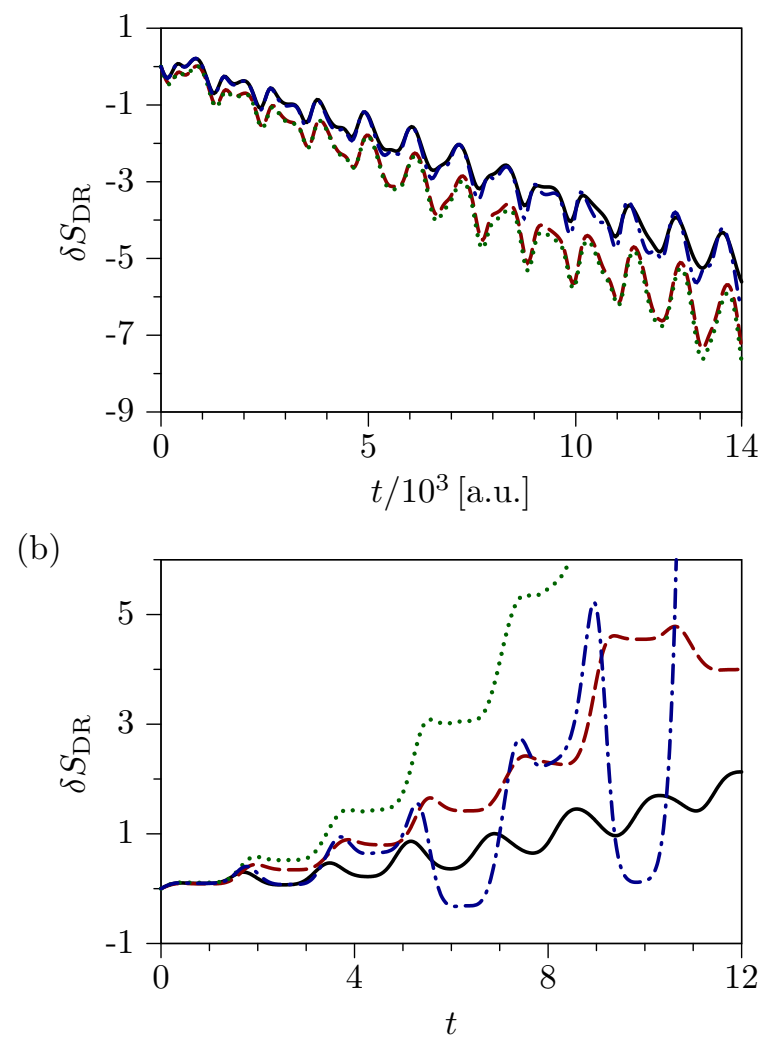

FIG. 7. Time dependence of the DR action difference $\delta S_{\mathrm{DR}}$ of Eq. 59 calculated for two neighboring trajectories (initial conditions $z^{0}$ and $w^{0}$ ). (a) Quartic oscillator (Fig. 8). (b) Collinear NCO molecule (Fig. 9). Delay times are as in Figs. 8 and $9, z^{0}$ is the phase-space center of the Gaussian initial state (of width $\sigma$ ) and $w^{0}=z^{0}+(\sigma, 0) / 2$. The order of the expansion (35) is distinguished by line type: "linear" (dashed), "quadratic" (dotted), and "linear $+1 / 2$ " (dashdotted). The symbol $1 / 2$ signifies that the derivatives of the stability matrix in Eq. B2 are neglected. Solid line shows numerically exact $\delta S_{\mathrm{DR}}$.

and CDRP are reliable only for short times since the prefactor 23) (understood as a function of time for fixed initial conditions) grows quickly and oscillates widely at later times.

\section{Collinear NCO molecule}

Typical chemical systems are neither globally harmonic as our pyrazine-based model, nor-fortunatelyas strongly chaotic as the quartic oscillator. In our last example we therefore consider a realistic, anharmonic system, in order to see how the CDR, DRP, and CDRP might perform in typical situations. For this purpose, we chose a two-dimensional model of the collinear NCO 

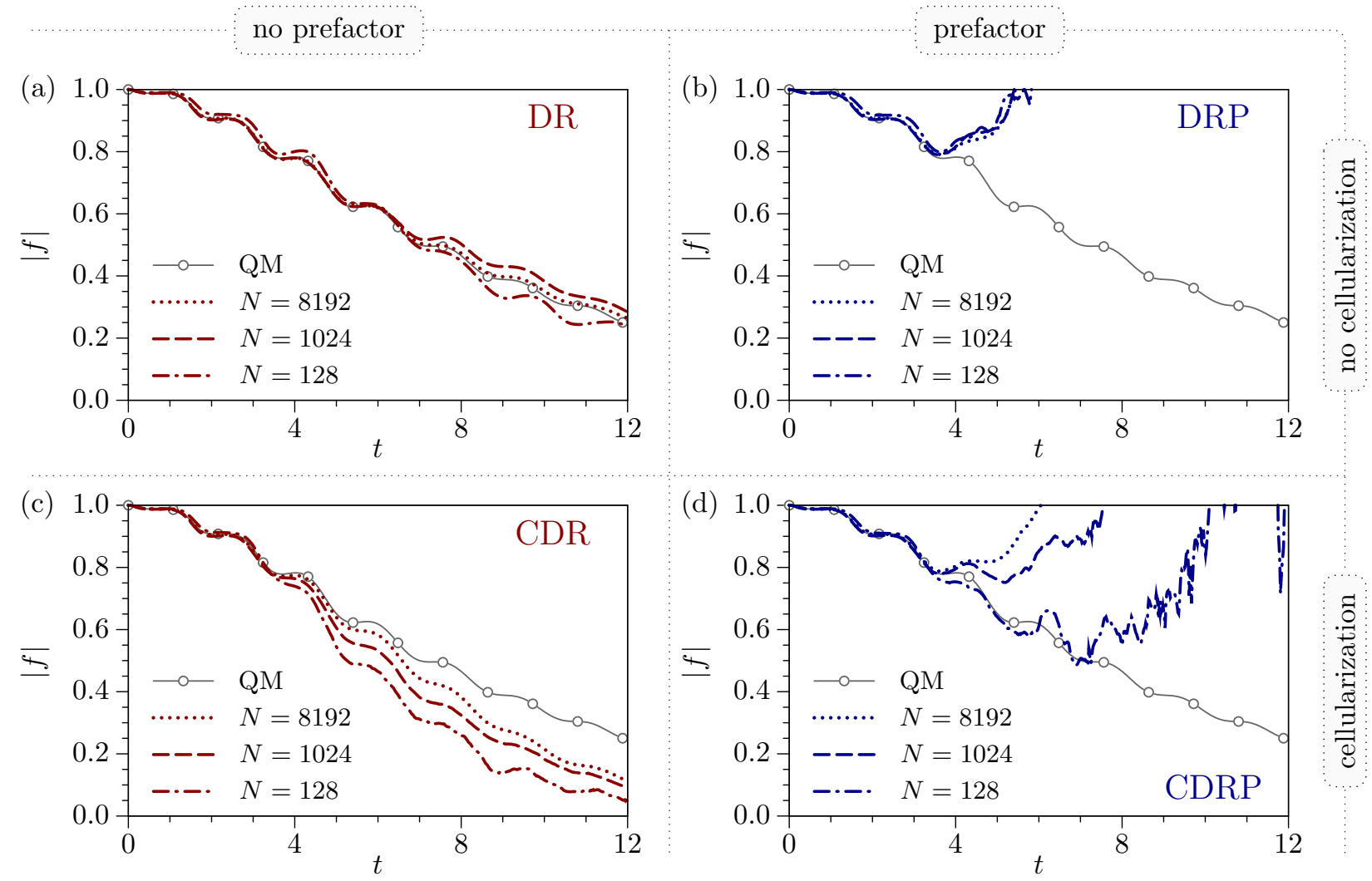

FIG. 8. Time correlation function for time-resolved stimulated emission in quartic oscillator (58) corresponding to zero time delay. Initial state is a Gaussian wave packet [Eq. (26)] with $\sigma_{1}=\sigma_{2}=1$ centered at $\left(Q_{\text {init }}, P_{\text {init }}\right)$, where $Q_{\text {init }}=(0,4)$ and $P_{\text {init }}=(4,0)$. Masses $m_{1}=m_{2}=1$ and the potential energy surfaces (58) are specified by $\beta_{0}=0.2$ and $\beta_{1}=0.2125$.

molecule based on the $\mathrm{X}^{2} \Pi$ (ground) and $\mathrm{A}^{2} \Sigma^{+}$(excited) PESs. ${ }^{51}$ The PESs are given in Ref. 51 in a form of a polynomial fitted to ab initio calculations on the domain $r_{1,2} \in[2,2.6]$ a.u. and $\theta \in\left[152^{\circ}, 208^{\circ}\right]$, specified in $r_{1}(\mathrm{~N}-\mathrm{C}), r_{2}(\mathrm{C}-\mathrm{O})$ bond-length coordinates and the bond angle $\theta$. We set $\theta=\pi$ (equilibrium value) and describe the reduced two-dimensional surfaces in the $r_{1}$ and $r_{2}$ coordinates by a simplified two-term form

$$
V\left(r_{1}, r_{2}\right)=V_{0}+\sum_{j=1,2} D_{j}\left\{1-\exp \left[-\beta_{j}\left(r_{j}-r_{j}^{\mathrm{e}}\right)\right]\right\}^{2},
$$

where the equilibrium bond lengths $r_{j}^{\mathrm{e}}$ are the same as in Ref. [51, while the parameters $V_{0}, D_{1,2}$, and $\beta_{1,2}$ were obtained by fitting potential (60) to the functional form of Ref. 51 on the domain $r_{j} \in\left[r_{j}^{\mathrm{e}}-\delta, r_{j}^{\mathrm{e}}+\delta\right]$ with $\delta=0.15$ a.u. Resulting values are summarized in Tab. [1. These parameters differ from the values employed in our earlier work ${ }^{819}$ and should better reflect the dynamics of this system. Frequency-mass-scaled normal mode coordinates of the $\mathrm{X}^{2} \Pi$ PES were used so that the vibrational ground state is in the harmonic approximation described by a Gaussian with unit widths centered at the origin.

The initial state for the TRSE calculation was prepared by the following procedure ${ }^{[52}$ First, we computed the $\mathrm{X}^{2} \Pi$ ground vibrational state by imaginary-time propagation. This state was then pumped to the $\mathrm{A}^{2} \Sigma^{+}$
TABLE I. Parameter $\AA^{\text {a }}$ of the collinear NCO model 60 .

\begin{tabular}{lccccccc}
\hline \hline & $V_{0}$ & $D_{1}$ & $\beta_{1}$ & $r_{1}^{\mathrm{e}}$ & $D_{2}$ & $\beta_{2}$ & $r_{2}^{\mathrm{e}}$ \\
\hline $\mathrm{X}^{2} \Pi$ & -167.653 & 0.150 & 1.698 & 2.302 & 0.333 & 1.160 & 2.246 \\
$\mathrm{~A}^{2} \Sigma^{+}$ & -167.549 & 0.144 & 1.984 & 2.234 & 0.398 & 1.140 & 2.232 \\
\hline
\end{tabular}

a All quantities are given in atomic units.

PES, propagated there for a net time of 520 a.u. $\approx$ $12.6 \mathrm{fs}$, dumped to $\mathrm{X}^{2} \Pi$, and propagated for additional 480 a.u. $\approx 11.6 \mathrm{fs}$. In order to facilitate computation of $C_{\Sigma}^{\rho_{W}}(z)$ in Eq. 28), we approximated the resulting state by a single Gaussian. An independent quantum calculation confirmed that this does not impact the spectrum significantly.

The TRSE correlation function for a delay time of $29 \mathrm{fs}$ is displayed in Fig. 9(a), confirming that the prefactor correction extends the agreement of the DR with the quantum correlation function to longer times. As a consequence, the prefactor correction yields sharper peaks in the corresponding spectrum, shown in Fig. 9(b). Finally, Fig. 9(c), comparing the statistical convergence of the DR, CDR, DRP, and CDRP, confirms that in NCO the cellularization increases numerical efficiency, al- 
though the effect is - as expected - smaller than in the harmonic pyrazine model [Fig. 6(c)].

\section{E. Computational details}

Classical trajectories needed in the DR, CDR, DRP, and CDRP were calculated with a fourth-order symplectic integrator, while quantum calculations employed the corresponding fourth-order split-operator method. 8 Time steps used for the pyrazine, quartic oscillator, and collinear NCO models were 0.5 a.u., $10^{-3}$, and 2.5 a.u., respectively. Also note that the branch of the square root in the prefactor in Eq. (37) was gradually adjusted in the course of the propagation in order to ensure that the phase of the prefactor be continuous in time.

\section{CONCLUSIONS}

We have introduced the CDRP, a rather accurate and efficient semiclassical method for computing ultrafast time-resolved electronic spectra. The CDRP is a two-stage refinement of the DR of fidelity amplitude: A prefactor correction, which typically increases accuracy, is followed by a cellularization procedure increasing efficiency (see Fig. 3). The new method has the same computational cost per trajectory as the two intermediate refinements, CDR and DRP; this cost is determined by propagating the stability matrix and its derivatives. While the cost per trajectory is significantly higher than the cost of each DR trajectory, the reduction in the required number of trajectories can in many situations result in higher efficiency compared with the DR.

The new methodology has been tested on several systems. In harmonic potentials (Figs. 4 and 5), pyrazinebased model (Fig. 6), and collinear NCO molecule (Fig. 9), the TRSE correlation functions and spectra computed with the CDRP were more accurate and required fewer trajectories than the corresponding quantities computed with the original DR. For harmonic potentials, analytical formulas have been derived; particularly, we have shown that cellularized calculations using a single trajectory are identical to the fully converged noncellular methods since the second-order expansion of the DR phase is exact. Moreover, in harmonic potentials the prefactor is the same for all trajectories. In contrast, in systems with highly nonlinear or chaotic dynamics, such as the quartic oscillator, the second-order approximation to the semiclassical action $S_{\mathrm{DR}}$ breaks down and its use can decrease both the accuracy and efficiency. Interestingly, in such systems the "bare" DR can perform rather well [see Fig. 8(a)], in agreement with previously published results. ${ }^{[7}$

An important result in its own right is the new simple, yet rigorous cellularization scheme for the DR, in which the size and the sampling weight of the Gaussian cells changes with their number. A similar cellularization
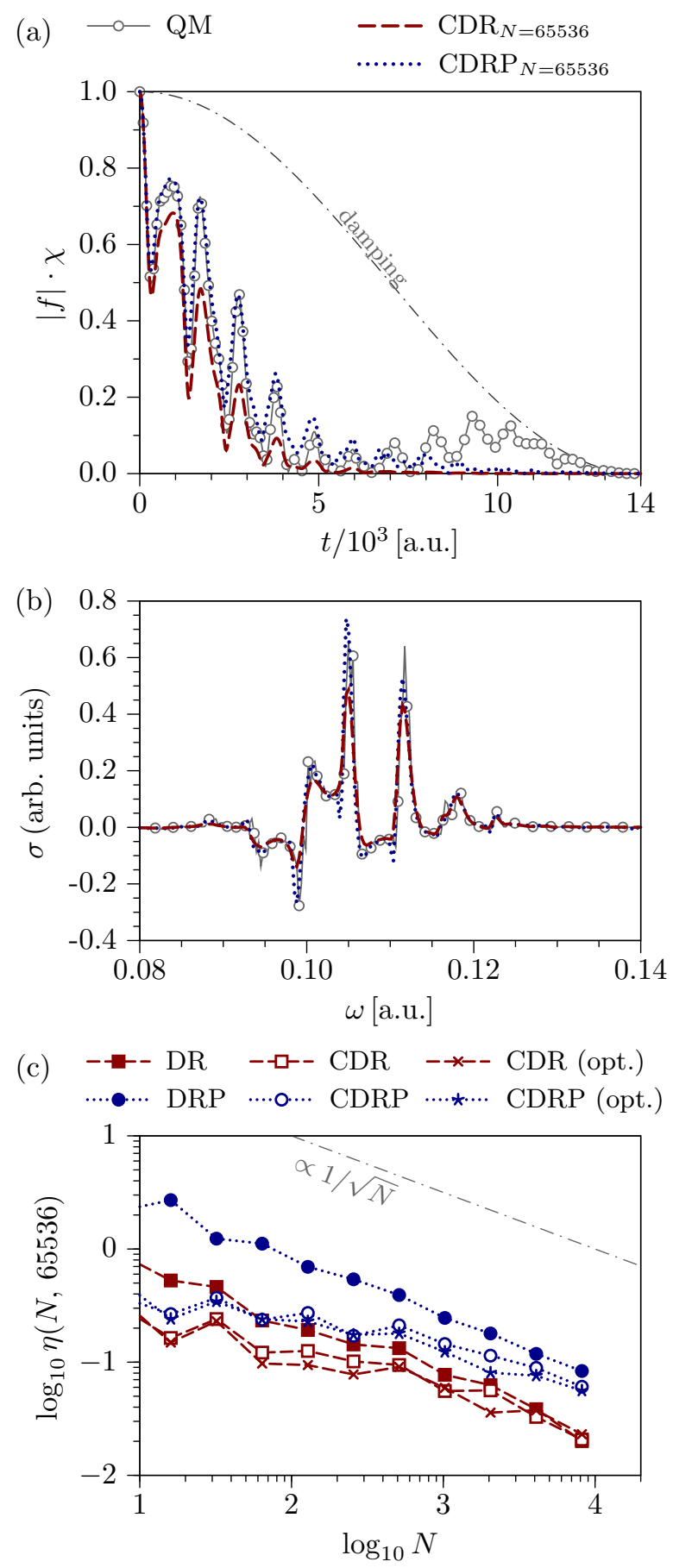

FIG. 9. Time-resolved stimulated emission in the NCO model of Subsec. IIID Initial state is a non-stationary state prepared by a pump-dump procedure ${ }^{9 \mid 52}$ discussed in the text, the delay time $\tau=1200 \mathrm{a} . \mathrm{u} . \approx 29 \mathrm{fs}$. (a) Time correlation function [damped by $\chi(t)$ of Eq. (56), shown as a dash-dotted line]. (b) Corresponding spectrum. (c) Convergence error $\eta$ [defined in Eq. (57)] of the damped correlation function as a function of the number of trajectories $N$. The points labeled by "opt." were computed with optimized expansion coefficients $C_{n}$ of Eq. (40) (see Subsec. II C). 
scheme using the inverse Weierstrass transform should be useful also for more general quantum dynamics using semiclassical initial value representations such as the Heller-Herman-Kluk-Kay propagator.

\section{ACKNOWLEDGMENTS}

This research was supported by the Swiss NSF with Grant No. 200021_124936/1 and NCCR MUST (Molecular Ultrafast Science \& Technology), and by the EPFL. We would like to thank M. Wehrle and T. Zimmermann for discussions.

\section{Appendix A: Phase-space propagator}

Semiclassical propagator in position representation, known as the Van Vleck propagator, is given by the expression

$$
\begin{aligned}
\left\langle q_{b}\left|e^{-i \hat{H} t / \hbar}\right| q_{a}\right\rangle_{\mathrm{SC}}=\sum_{\substack{j \\
q_{a} \rightsquigarrow q_{b}}}( & 2 i \pi \hbar)^{-D / 2} \operatorname{det}\left(\frac{\partial^{2} S_{j}}{\partial q_{a} \partial q_{b}}\right)^{-1 / 2} \\
& \times e^{i S_{j}\left(q_{a}, q_{b} ; t\right) / \hbar-i \nu_{j} \pi / 2}, \quad(\mathrm{~A} 1)
\end{aligned}
$$

where the summation is performed over all trajectories $j$ of the classical Hamiltonian $H$ starting from $q_{a}$ and arriving at $q_{b}$ after time $t, S_{j}$ is the classical action along the $j$ th path, and $\nu_{j}$ is its Morse index.

The phase-space propagator is the Wigner transform of the evolution operator,

$$
U_{W}(x, t)=\int d^{D} s\left\langle q-s / 2\left|e^{-i \hat{H} t / \hbar}\right| q+s / 2\right\rangle e^{i s^{\top} \cdot p / \hbar} .
$$

The integrand in the last equation includes the position propagator between $q+s / 2$ to $q-s / 2$. Using the Van Vleck propagator, we can obtain the semiclassical expression for Eq. A2]

$$
U_{\mathrm{SC}}(\bar{x}, t)=2^{D} \sum_{j}\left|\operatorname{det}\left(I+M_{j}^{t}\right)\right|^{-1 / 2} \exp \left[\frac{i}{\hbar} S_{\mathrm{c}, j}(\bar{x}, t)\right] \text {, }
$$

where the sum runs over all paths $j$ centered at $\bar{x}$, i.e., paths for which $\left(x^{0}+x^{t}\right) / 2=\bar{x}$ [see Fig. 10, $M^{t}$ is the stability matrix of the flow $x^{0} \rightarrow x^{t}$, and the function $S_{\mathrm{c}}(\bar{x}, t)$, called center-action, is defined as

$$
S_{\mathrm{c}}(\bar{x}, t)=\oint p^{\top} \cdot d q-\int_{0}^{t} H\left(x^{t^{\prime}}, t^{\prime}\right) d t^{\prime},
$$

where the first term is the symplectic area enclosed by a closed path consisting of a trajectory centered at $\bar{x}$ and the chord connecting this trajectory's final and initial points.

In general, the center-action is multivalued and each of its branches is associated with a classical trajectory

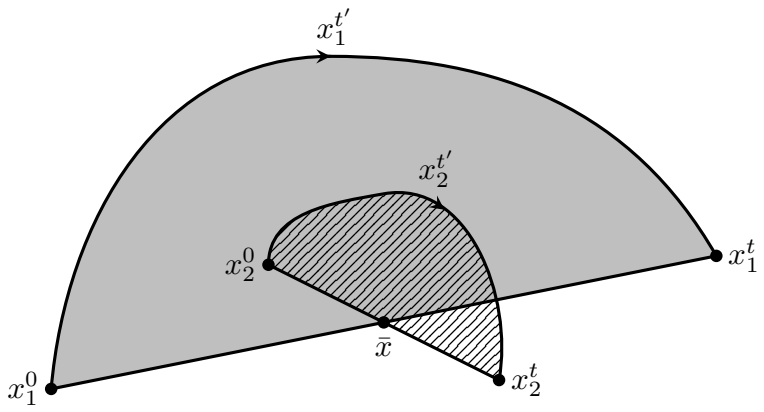

FIG. 10. Geometrical interpretation of the semiclassical phase space propagator. Two trajectories $\left(x_{1}^{t^{\prime}}\right.$ and $\left.x_{2}^{t^{\prime}}\right)$ contributing to $U_{\mathrm{SC}}(\bar{x}, t)$ are shown; $\bar{x}$ is the midpoint of both. Geometrical parts of the center-actions $S_{\mathrm{c}, 1}$ and $S_{\mathrm{c}, 2}$ are displayed as filled and hatched areas, respectively.

centered at $\bar{x}$, as shown in Fig. 10 . The stability matrix, defined as $M^{t}:=\partial x^{t} / \partial x^{0}$, defines the local linearization of the classical path in the tangent phase-space and the phase-space propagator has caustics whenever $M^{t}$ has an eigenvalue $-1 . \sqrt{35}$ Moreover, $M^{t}$ is equal to the Cayley transform of $\frac{1}{2} J \cdot \partial^{2} S_{\mathrm{c}}(\bar{x}, t) / \partial \bar{x}^{2}$,

$$
M^{t}=\left(I-\frac{J}{2} \cdot \frac{\partial^{2} S_{c}}{\partial \bar{x}^{2}}\right) \cdot\left(I+\frac{J}{2} \cdot \frac{\partial^{2} S_{c}}{\partial \bar{x}^{2}}\right)^{-1}
$$

and the determinant in phase-space propagator $\mathrm{A} 3$ can be written in terms of the center action as 36

$$
2^{2 D}\left[\operatorname{det}\left(I+M^{t}\right)\right]^{-1}=\operatorname{det}\left(I+\frac{J}{2} \cdot \frac{\partial^{2} S_{\mathrm{c}}}{\partial \bar{x}^{2}}\right) .
$$

The last relation follows from the fact that both $M^{t}$ and the Hessian of $S_{c}(\bar{x}, t)$ define the same local linearization of the classical equations of motion in a neighborhood of a classical trajectory $x^{t^{\prime}}$. This linearization is described by the mapping:

$$
x^{0}=\bar{x}+\frac{J}{2} \cdot \frac{\partial S_{\mathrm{c}}}{\partial \bar{x}} \rightarrow x^{t}=\bar{x}-\frac{J}{2} \cdot \frac{\partial S_{\mathrm{c}}}{\partial \bar{x}} .
$$

For short times, the Wigner transformation $\mathcal{E}_{W}(x, t)$ of the echo operator can be approximated by a propagator A3 with a single classical trajectory, 24/36 as in Eq. 18.

\section{Appendix B: Derivatives of the DR phase in Eq. (35)}

One of the main numerical prerequisites of both the DRP and CDR is the second order expansion of the DR phase, $S_{\mathrm{DR}}\left(x^{0}, t\right)$, as indicated in Eq. (35). Here we describe a symplectic numerical procedure for obtaining the time derivatives of the phase-space derivatives $\partial^{|\alpha|} S_{\mathrm{DR}}\left(x^{0}, t\right) / \partial\left(x^{0}\right)^{\alpha}$ for $|\alpha| \leq 2$ (multi-index notation was used). 
As in other semiclassical methods, the knowledge of the Hessian of the potential is required for propagating the stability matrix $M^{t}$. Below we show that in order to obtain the Hessian of $S_{\mathrm{DR}}\left(x^{0}, t\right)$ with respect to $x^{0}$, third derivatives of the potential, $\nabla^{3} V$, are also needed. Although the third derivative is in principle required also in Cellular Dynamics 23 and Cellularized Frozen Gaussian approximation, 38 the associated computational cost has led the authors of these methods to neglect the contribution of terms depending on $\nabla^{3} V$. However, as demonstrated in Fig. 7(a), this contribution can be essential even in simple realistic models such as the collinear NCO molecule.

First, consider components of the gradient of $S_{\mathrm{DR}}$,

$$
\frac{\partial S_{\mathrm{DR}}}{\partial x_{j}^{0}}=\int_{0}^{t} d t^{\prime} \frac{\partial \Delta H}{\partial x_{k}^{t^{\prime}}} \frac{\partial x_{k}^{t^{\prime}}}{\partial x_{j}^{0}}=-\int_{0}^{t} d t^{\prime} \Delta F_{k}^{t^{\prime}} M_{k j}^{t^{\prime}},
$$

where $\Delta H=H_{2}-H_{1}, \Delta F^{t} \equiv-\partial \Delta H / \partial x^{t}$ is the force difference vector, and repeated indexes imply summation. Similarly, the components of the Hessian of $S_{\mathrm{DR}}$ are

$$
\frac{\partial^{2} S_{\mathrm{DR}}}{\partial x_{i}^{0} \partial x_{j}^{0}}=\int_{0}^{t} d t^{\prime}\left(\Delta \mathcal{H}_{k s}^{t^{\prime}} M_{k i}^{t^{\prime}} M_{s j}^{t^{\prime}}-\Delta F_{k}^{t^{\prime}} N_{k, i j}^{t^{\prime}}\right),
$$

where $\Delta \mathcal{H}^{t}$ denotes the Hessian of $\Delta H$ at time $t$ and

$$
N_{k, i j}^{t}:=\frac{\partial^{2} x_{k}^{t}}{\partial x_{i}^{0} \partial x_{j}^{0}}=\frac{\partial}{\partial x_{i}^{0}} M_{k j}^{t} .
$$

While the time integrals in Eqs. (B1) and (B2) are evaluated using composite Newton-Cotes formulas, the integrands can be propagated symplectically. The algorithm for $N^{t}$ propagation, e.g., is obtained by applying the chain rule to the preceding equation,

$$
N_{k, i j}^{t+\delta t}=\frac{\partial x_{k}^{t+\delta t}}{\partial x_{s}^{t}} N_{s, i j}^{t}+\frac{\partial^{2} x_{k}^{t+\delta t}}{\partial x_{n}^{t} \partial x_{s}^{t}} M_{n i}^{t} M_{s j}^{t},
$$

whereas the symplectic propagation scheme for the stability matrix was described previously!

$$
M_{i j}^{t+\delta t}=\frac{\partial x_{i}^{t+\delta t}}{\partial x_{k}^{t}} M_{k j}^{t} .
$$

Derivatives of phase-space coordinates in Eqs. (B4) and (B5) are obtained from symplectic integrators for $q$ and $p$, which are for standard Hamiltonians of the form $\sum_{i} p_{i}^{2} / 2 m_{i}+V(q)$ based on a Lie-Trotter-type ${ }^{54}$ decomposition of a short-time propagator into elementary steps within which the system is propagated under the influence of either the kinetic or the potential term only. Action of the kinetic term $\sum_{i} p_{i}^{2} / 2 m_{i}$ for time $\delta t$ results in a phase-space shear preserving the momentum,

$$
\left(q^{t+\delta t}, p^{t+\delta t}\right)=\left(q^{t}+m^{-1} \cdot p^{t} \delta t, p^{t}\right),
$$

whereas the action of the potential term $V(q)$ changes momentum and preserves position:

$$
\left(q^{t+\delta t}, p^{t+\delta t}\right)=\left(q^{t}, p^{t}-\frac{\partial V\left(q^{t}\right)}{\partial q^{t}} \delta t\right) .
$$

Since the only nonlinear dependence of $\left(q^{t+\delta t}, p^{t+\delta t}\right)$ on $\left(q^{t}, p^{t}\right)$ stems from the presence of the potential gradient in Eq. (B7), the second derivative terms in Eq. (B4) are nonzero only during the "p-propagation" (B7) and explicitly involve derivatives of the Hessian:

$$
\frac{\partial^{2} p_{k}^{t+\delta t}}{\partial q_{i}^{t} \partial q_{j}^{t}}=-\delta t \frac{\partial^{3} V\left(q^{t}\right)}{\partial q_{k}^{t} \partial q_{i}^{t} \partial q_{j}^{t}}
$$

As already mentioned, these third derivatives of the potential, which should appear in other semiclassical propagation schemes ${ }^{23 \mid 38}$ as well, are usually neglected in order to reduce computational cost. Yet, in Section IIID we have shown that they can play an essential role even in rather simple systems such as the NCO.

\section{Appendix C: Complex conjugate of Eq. 22}

As discussed in Subsec. II B switching the roles of the PESs in Eq. (4) for the correlation function corresponds (for $\tau=0$ ) to taking the complex conjugate of this equation. Likewise, when one interchanges the PESs, the DR phase and hence the matrix $B_{x^{0}}^{t}$ change the sign. However, since the prefactor $A_{\mathrm{DRP}}$ in Eq. 22 is real, it might seem that the DRP is incompatible with this operation.

Here we demonstrate that this is not the case by proving that $\operatorname{det}\left(I+J \cdot B_{x^{0}}^{t}\right)=\operatorname{det}\left(I-J \cdot B_{x^{0}}^{t}\right)$. To this end, consider a general, symmetric, $2 D \times 2 D$ matrix $A$ and let $a$ denote any of the eigenvalues of $J \cdot A$. Then

$$
\begin{aligned}
0 & =\operatorname{det}(J \cdot A-a I)=\operatorname{det}\left(A-a J^{\top}\right) \\
& =\operatorname{det}(A+a J)=\operatorname{det}(A+a J)^{\top} \\
& =\operatorname{det}\left(A+a J^{\top}\right)=\operatorname{det}(J \cdot A+a I),
\end{aligned}
$$

where we have used the properties $-J=J^{\top}=J^{-1}$, $\operatorname{det} J=1$, and that taking the transpose of an arbitrary square matrix does not affect its determinant.

Equation (C1) shows that the eigenvalues of $J \cdot A$ come in pairs $(a,-a)$. This directly implies that $\operatorname{det}(I+J \cdot A)=$ $\operatorname{det}(I-J \cdot A)$. [This also follows from setting $a=1$ in Eq. (C1) and using the fact that the matrix $J \cdot A$ is of even order.]

${ }^{1}$ C. Z. Bisgaard, O. J. Clarkin, G. Wu, A. M. D. Lee, O. Gessner, C. C. Hayden, and A. Stolow, Science 323, 1464 (2009) C. Bressler, C. Milne, V.-T. Pham, A. ElNahhas, R. M. van der Veen, W. Gawelda, S. Johnson, P. Beaud, D. Grolimund, M. Kaiser, C. N. Borca, G. Ingold, R. Abela, and M. Chergui, Science 323, 489 (2009) F. Carbone, O.-H. Kwon, and A. H. Zewail, Science 325, 181 (2009)

${ }^{2}$ W. H. Miller, J. Chem. Phys. 53, 3578 (1970)

${ }^{3}$ E. J. Heller, J. Chem. Phys. 75, 2923 (1981)

${ }^{4}$ M. F. Herman and E. Kluk, Chem. Phys. 91, 27 (1984)

${ }^{5}$ W. H. Miller, J. Phys. Chem. 105, $2942(2001)$.

${ }^{6}$ M. F. Herman, Annu. Rev. Phys. Chem. 45, 83 (1994), M. Thoss and H. Wang, Annu. Rev. Phys. Chem. 55, 299 (2004) K. G. Kay, Annu. Rev. Phys. Chem. 56, 255 (2005) M. Ceotto, Y. Zhuang, and W. L. Hase, J. Chem. Phys. 138, 054116 (2013)

${ }^{7}$ J. Vaníček, Phys. Rev. E 70, 055201 (2004) Phys. Rev. E 73, 046204 (2006) 
${ }^{8}$ M. Wehrle, M. Šulc, and J. Vaníček, Chimia 65, 334 (2011)

${ }^{9}$ M. Šulc and J. Vaníček, Mol. Phys. 110, $945(2012)$

${ }^{10}$ J. Vaníček and E. J. Heller, Phys. Rev. E 68, 056208 (2003)

${ }^{11}$ W. H. Miller and F. T. Smith, Phys. Rev. A 17, 939 (1978) L. M. Hubbard and W. H. Miller, J. Chem. Phys. 78, 1801 (1983)

${ }^{12}$ S. Mukamel, J. Chem. Phys. 77, 173 (1982) Principles of nonlinear optical spectroscopy, 1st ed. (Oxford University Press, New York, 1999).

${ }^{13}$ N. E. Shemetulskis and R. F. Loring, J. Chem. Phys. 97, 1217 (1992)

${ }^{14}$ J. M. Rost, J. Phys. B 28, L601 (1995)

${ }^{15}$ H. Wang, X. Sun, and W. H. Miller, J. Chem. Phys. 108, 9726 (1998)

10 Z. Li, J.-Y. Fang, and C. C. Martens, J. Chem. Phys. 104, 6919 (1996); S. A. Egorov, E. Rabani, and B. J. Berne, J. Chem. Phys. 108, 1407 (1998) J. Chem. Phys. 110, 5238 (1999)

${ }^{17}$ Q. Shi and E. Geva, J. Chem. Phys. 122, 064506 (2005)

${ }^{18}$ J. A. Poulsen, G. Nyman, and P. J. Rossky, J. Chem. Phys. 119, 12179 (2003)

${ }^{19}$ S. Bonella and D. F. Coker, J. Chem. Phys. 122, 194102 (2005)

${ }^{20}$ T. Zimmermann and J. Vaníček, J. Chem. Phys. 136, 094106 (2012); J. Chem. Phys. 137, 22A516 (2012)

${ }^{21}$ W. Wang, G. Casati, B. Li, and T. Prosen, Phys. Rev. E 71, $037202(2005)$ N. Ares and D. A. Wisniacki, Phys. Rev. E 80, 046216 (2009) D. A. Wisniacki, N. Ares, and E. G. Vergini, Phys. Rev. Lett. 104, 254101 (2010) I. García-Mata and D. A. Wisniacki, J. Phys. A 44, 315101 (2011)

${ }^{22}$ C. Mollica and J. Vaníček, Phys. Rev. Lett. 107, 214101 (2011)

${ }^{23}$ E. J. Heller, J. Chem. Phys. 94, 2723 (1991)

${ }^{24}$ E. Zambrano and A. M. Ozorio de Almeida, Phys. Rev. E 84, 045201(R) (2011)

${ }^{25}$ W. T. Pollard, S.-Y. Lee, and R. A. Mathies, J. Chem. Phys. 92, 4012 (1990)

${ }^{26}$ G. Stock and W. Domcke, Phys. Rev. A 45, 3032 (1992)

${ }^{27} \mathrm{D}$. J. Tannor, Introduction to Quantum Mechanics: A TimeDependent Perspective (University Science Books, 2004).

${ }^{28}$ T. Gorin, T. Prosen, T. H. Seligman, and M. Žnidarič, Phys. Rep. 435, 33 (2006) P. Jacquod and C. Petitjean, Adv. Phys. $\mathbf{5 8}, 67(2009)$

${ }^{29}$ H. M. Pastawski, P. R. Levstein, G. Usaj, J. Raya, and J. Hirschinger, Physica A 283, 166 (2000)

${ }^{30}$ F. M. Cucchietti, D. A. R. Dalvit, J. P. Paz, and W. H. Zurek, Phys. Rev. Lett. 91, 210403 (2003) T. Gorin, T. Prosen, and T. H. Seligman, New J. Phys. 6, 20 (2004)

${ }^{31}$ C. Petitjean, D. V. Bevilaqua, E. J. Heller, and P. Jacquod,
Phys. Rev. Lett. 98, 164101 (2007)

${ }^{32}$ T. Zimmermann and J. Vaníček, J. Chem. Phys. 132, 241101 (2010)

${ }^{33}$ B. Li, C. Mollica, and J. Vaníček, J. Chem. Phys. 131, 041101 (2009)

${ }^{34}$ T. Zimmermann, J. Ruppen, B. Li, and J. Vaníček, Int. J. Quant. Chem. 110, 2426 (2010)

${ }^{55}$ M. V. Berry, Proc. Roy. Soc. London Sect. A 423, 219 (1989)

${ }^{36}$ A. M. Ozorio de Almeida, Phys. Rep. 295, 265 (1998)

${ }^{37}$ O. Bohigas, M.-J. Giannoni, A. M. O. de Almeida, and C. Schmit, Nonlinearity 8, 203 (1995)

${ }^{38}$ A. R. Walton and D. E. Manolopoulos, Mol. Phys. 87, 961 (1996)

${ }^{39}$ V. S. Filinov, Nucl. Phys. B 271, 717 (1986)

${ }^{40}$ N. Makri and W. H. Miller, Chem. Phys. Lett. 139, 10 (1987)

${ }^{41}$ H. Wang, D. E. Manolopoulos, and W. H. Miller, J. Chem. Phys. 115, $6317(2001)$

${ }^{42}$ D. V. Widder, Bull. Amer. Math. Soc. 60, 444 (1954)

${ }^{43}$ J. Doll, D. Freeman, and M. Gillan, Chem. Phys. Lett. 143, 277 (1988)

${ }^{44}$ W. H. Press, S. A. Teukolsky, W. T. Vetterling, and B. P. Flannery, Numerical Recipes, 3rd ed. (Cambridge University Press, 2007).

${ }^{45}$ J. Nocedal and S. J. Wright, Numerical optimization, 2nd ed. (Springer, 2006).

${ }^{46}$ X. Sun and W. H. Miller, J. Chem. Phys. 110, 6635 (1999)

${ }^{47}$ G. Stock, C. Woywod, W. Domcke, T. Swinney, and B. S. Hudson, J. Chem. Phys. 103, 6851 (1995)

${ }^{48}$ F. Duschinsky, Acta Physicochim. URSS 7, 551 (1937); İ. Özkan, J. Mol. Spec. 139, 147 (1990)

${ }^{49}$ H.-D. Meyer, F. Gatti, and G. A. Worth, eds., Multidimensional Quantum Dynamics: MCTDH Theory and Applications, 1st ed. (Wiley-VCH, Weinheim, 2009).

${ }^{50}$ H.-D. Meyer, J. Chem. Phys. 84, 3147 (1986) R. L. Waterland, J.-M. Yuan, C. C. Martens, R. E. Gillilan, and W. P. Reinhardt, Phys. Rev. Lett. 61, 2733 (1988) B. Eckhardt, G. Hose, and E. Pollak, Phys. Rev. A 39, 3776 (1989) O. Bohigas, S. Tomsovic, and D. Ullmo, Phys. Rep. 223, 43 (1993) F. Revuelta, E. G. Vergini, R. M. Benito, and F. Borondo, Phys. Rev. E 85, $026214(2012)$

${ }^{51}$ Y. Li, S. Carter, G. Hirsch, and R. J. Buenker, Mol. Phys. 80, 145 (1993)

${ }^{52}$ R. Baer and R. Kosloff, J. Phys. Chem. 99, 2534 (1995).

${ }^{53}$ M. L. Brewer, J. S. Hulme, and D. E. Manolopoulos, J. Chem.

Phys. 106, 4832 (1997)

${ }^{54}$ H. F. Trotter, Proc. Amer. Math. Soc. 10, 545 (1959) 